\title{
A new extreme value copula and new families of univariate distributions based on Freund's exponential model
}

https://doi.org/10.1515/demo-2020-0018

Received March 17, 2020; accepted October 23, 2020

\begin{abstract}
The use of the exponential distribution and its multivariate generalizations is extremely popular in lifetime modeling. Freund's bivariate exponential model (1961) is based on the idea that the remaining lifetime of any entity in a bivariate system is shortened when the other entity defaults. Such a model can be quite useful for studying systemic risk, for instance in financial systems. Guzmics and Pflug (2019) revisited Freund's model, deriving the corresponding bivariate copula and examined some characteristics of it; furthermore, we opened the door for a multivariate setting. Now we present further investigations in the bivariate model: we compute the tail dependence coefficients, we examine the marginal and joint distributions of the componentwise maxima, which leads to an extreme value copula, which - to the best of our knowledge - has not been investigated in the literature yet. The original bivariate model of Freund has been extended to more variables by several authors. We also turn to the multivariate setting, and our focus is different from that of the previous generalizations, and therefore it is novel: examining the distribution of the sum and of the average of the lifetime variables (provided that the shock parameters are all the same) leads to new families of univariate distributions, which we call Exponential Gamma Mixture Type I and Type II (EGM) distributions. We present their basic properties, we provide asymptotics for them, and finally we also provide the limiting distribution for the EGM Type II distribution.
\end{abstract}

Keywords: Lifetime modeling, multivariate distributions, copulas, Freund copula, extreme value copulas, sums of non-independent random variables, parametric univariate distributions

MSC: 60E05, 60G70, 62H05

\section{Introduction}

We consider the bivariate lifetime model introduced by Freund [4]. The idea is that the lifetimes of two entities (we also refer to them as "institutions") are originally assumed to be $\operatorname{Exp}\left(\lambda_{i}\right)$ distributed $(i=1,2)$, and when one of the entities defaults, it modifies the remaining lifetime of the other entity by increasing the intensity of its original exponential lifetime. This assumption is a possible way for modelling cascading effects when we examine systemic risk in finance. The construction in detail looks as follows. (We recall Section 2.1 from Guzmics and Pflug [6].)

Let $Y_{i} \sim \operatorname{Exp}\left(\lambda_{i}\right)(i=1,2)$ be independent random variables. They are attributed as auxiliary lifetime variables (if one wishes as pre-lifetime variables) of the two entities of the system. When in a certain realization the first entity defaults earlier, i.e., $Y_{1}<Y_{2}$, then the second entity will continue its operation according

\footnotetext{
^Corresponding Author: Sándor Guzmics: University of Vienna. Department of Statistics and Operations Research (DSOR), Oskar Morgenstern Platz 1, A-1090 Wien-Vienna, Austria, E-mail: sandor.guzmics@univie.ac.at Georg Ch. Pflug: DSOR and International Institute for Applied Systems Analysis (IIASA), Laxenburg, Austria, E-mail: georg.pflug@univie.ac.at
} 
to another exponentially distributed random variable $Z_{2} \sim \operatorname{Exp}\left(\lambda_{2}+a_{2}\right)$, which is independent of $Y_{1}$ and $Y_{2}$. The parameter $a_{2} \geq 0$ is called the shock parameter, and it expresses the effect of the default of the first institution on the second institution. $Z_{1}$ is defined analogously: when $Y_{2}<Y_{1}$, then $Z_{1} \sim \operatorname{Exp}\left(\lambda_{1}+a_{1}\right)$, where $a_{1} \geq 0$ is a shock parameter.

The actual lifetime variables of the two entities are denoted by $X_{1}, X_{2}$, and - in the light of the above construction - can be written as follows.

$$
\begin{aligned}
& \text { If } Y_{1}<Y_{2} \text {, then }\left\{\begin{array}{l}
X_{1}:=Y_{1}, \\
X_{2}:=Y_{1}+Z_{2}, \text { where } Z_{2} \sim \operatorname{Exp}\left(\lambda_{2}+a_{2}\right) \text { independent of } Y_{1}, Y_{2} .
\end{array}\right. \\
& \text { If } Y_{2}<Y_{1} \text {, then }\left\{\begin{array}{l}
X_{2}:=Y_{2}, \\
X_{1}:=Y_{2}+Z_{1}, \text { where } Z_{1} \sim \operatorname{Exp}\left(\lambda_{1}+a_{1}\right) \text { independent of } Y_{1}, Y_{2} .
\end{array}\right.
\end{aligned}
$$

The new lifetime variables $X_{1}, X_{2}$ can be expressed explicitly in terms of $Y_{1}, Y_{2}, Z_{1}, Z_{2}$ :

$$
\left\{\begin{array}{l}
X_{1}=Y_{1} \cdot \mathbb{1}_{\left\{Y_{1}<Y_{2}\right\}}+\left(Y_{2}+Z_{1}\right) \cdot \mathbb{1}_{\left\{Y_{2}<Y_{1}\right\}}, \\
X_{2}=Y_{2} \cdot \mathbb{1}_{\left\{Y_{2}<Y_{1}\right\}}+\left(Y_{1}+Z_{2}\right) \cdot \mathbb{1}_{\left\{Y_{1}<Y_{2}\right\}} .
\end{array}\right.
$$

The case $Y_{1}=Y_{2}$ does not need to be taken into account, since it has probability zero.

The resulting bivariate distribution was first presented in [4], and was investigated further in [6]. The joint cumulative distribution function (cdf) of the resulting lifetime variables $\left(X_{1}, X_{2}\right)$ (if $\lambda_{1} \neq a_{2}$ and $\left.\lambda_{2} \neq a_{1}\right)$ is given by

$$
H(x, y)=\left\{\begin{array}{ccc}
1+\frac{\lambda_{1}}{\lambda_{1}-a_{2}} \cdot e^{-\left(\lambda_{1}-a_{2}\right) x} \cdot e^{-\left(\lambda_{2}+a_{2}\right) y}+\frac{a_{1}}{\lambda_{2}-a_{1}} \cdot e^{-\left(\lambda_{1}+\lambda_{2}\right) x}- & \\
\frac{\lambda_{2}}{\lambda_{2}-a_{1}} \cdot e^{-\left(\lambda_{1}+a_{1}\right) x}-\frac{\lambda_{1}}{\lambda_{1}-a_{2}} \cdot e^{-\left(\lambda_{2}+a_{2}\right) y}, & \text { if } & 0 \leq x \leq y, \\
1+\frac{\lambda_{2}}{\lambda_{2}-a_{1}} \cdot e^{-\left(\lambda_{2}-a_{1}\right) y} \cdot e^{-\left(\lambda_{1}+a_{1}\right) x}+\frac{a_{2}}{\lambda_{1}-a_{2}} \cdot e^{-\left(\lambda_{1}+\lambda_{2}\right) y}- & \\
\frac{\lambda_{1}}{\lambda_{1}-a_{2}} \cdot e^{-\left(\lambda_{2}+a_{2}\right) y}-\frac{\lambda_{2}}{\lambda_{2}-a_{1}} \cdot e^{-\left(\lambda_{1}+a_{1}\right) x}, & \text { if } & 0 \leq y \leq x .
\end{array}\right.
$$

The marginal cdf of $X_{1}$ (if $\lambda_{2} \neq a_{1}$ ) and of $X_{2}$ (if $\lambda_{1} \neq a_{2}$ ) are given by

$$
\begin{array}{ll}
F(x)=1-\frac{\lambda_{2}}{\lambda_{2}-a_{1}} \cdot e^{-\left(\lambda_{1}+a_{1}\right) x}+\frac{a_{1}}{\lambda_{2}-a_{1}} \cdot e^{-\left(\lambda_{1}+\lambda_{2}\right) x}, & x \geq 0, \\
G(y)=1-\frac{\lambda_{1}}{\lambda_{1}-a_{2}} \cdot e^{-\left(\lambda_{2}+a_{2}\right) y}+\frac{a_{2}}{\lambda_{1}-a_{2}} \cdot e^{-\left(\lambda_{1}+\lambda_{2}\right) y}, & y \geq 0 .
\end{array}
$$

For the remaining parameter constellations and for the joint and marginal densities, we refer again to [4] and [6]. Here we only highlight the special case $\lambda_{1}=\lambda_{2}=a_{1}=a_{2}=1$, which will become important in some of the upcoming computations; in this case the marginal cdfs are

$$
F(x)=1-(1+x) \cdot e^{-2 x}, x \geq 0, \quad G(y)=1-(1+y) \cdot e^{-2 y}, y \geq 0 .
$$

We will also need the copula $C(u, v)$ of the lifetime variables $X_{1}$ and $X_{2}$. It was explained in [6] that the copula function cannot be expressed explicitly in terms of $u$ and $v$, because the inverse of the strictly increasing marginal cdfs $F$ and $G$ given in (4) and (5) cannot be expressed explicitly. So we provide a semiexplicit form for the parameter setting $\lambda_{1}=\lambda_{2}=1, a_{1}=a_{2}=a<\infty$, which will be used in the subsequent computations.

$$
\begin{gathered}
C^{(a)}(u, v)=H\left(F^{-1}(u), G^{-1}(v)\right)= \\
=\left\{\begin{array}{ccc}
1+\frac{1}{1-a} \cdot e^{-(1-a) F^{-1}(u)} \cdot e^{-(1+a) G^{-1}(v)}+\frac{a}{1-a} \cdot e^{-2 F^{-1}(u)}- & \\
\frac{1}{1-a} \cdot e^{-(1+a) F^{-1}(u)}-\frac{1}{1-a} \cdot e^{-(1+a) G^{-1}(v)}, & \text { if } & 0 \leq F^{-1}(u) \leq G^{-1}(v), \\
1+\frac{1}{1-a} \cdot e^{-(1-a) G^{-1}(v)} \cdot e^{-(1+a) F^{-1}(u)}+\frac{a}{1-a} \cdot e^{-2 G^{-1}(v)}- & \\
\frac{1}{1-a} \cdot e^{-(1+a) G^{-1}(v)}-\frac{1}{1-a} \cdot e^{-(1+a) F^{-1}(u)}, & \text { if } & 0 \leq G^{-1}(v) \leq F^{-1}(u) .
\end{array}\right.
\end{gathered}
$$


Notice that the four parameters can be written as a matrix

$$
A=\left(\begin{array}{cc}
\lambda_{1} & a_{1} \\
a_{2} & \lambda_{2}
\end{array}\right)
$$

so we will use the notation Freund $(A)$, when we refer to the distribution given in (3). The same notation Freund $(A)$ and the analogue notation Freund $(a, n)$ will be used in Section 3 for the analogous situations in the multivariate case $(n \geq 2)$. The model also allows to set $a_{1}=\infty$ or $a_{2}=\infty$, which means that the default of one institution causes the immediate default of the other one. For instance, if $a_{1}=a_{2}=\infty$, then the underlying lifetimes variables $X_{1}, X_{2}$ are completely dependent, and the copula function in (7) reduces to $C^{(\infty)}(u, v)=\min \{u, v\}$, i.e., the Fréchet upper bound. In some situations we will focus on the special parameter setting $\lambda_{1}=\lambda_{2}=1, a_{1}=a_{2}=a \geq 0$, which will be denoted by $\operatorname{Freund}(a, 2)$. In accordance with this, we can speak about the $\operatorname{Freund}(A)$ and $\operatorname{Freund}(a, 2)$ copulas. This latter one is given in (7).

The paper is organized as follows. In Section 2, we deal with the bivariate model, examine the relation between componentwise maxima and provide a new extreme value copula. In Section 3, we present the multivariate setting and investigate the sum and the average of the lifetime variables. This setting leads to two new, closely related families of univariate distributions, which we call Exponential Gamma Mixture Type I and Type II distributions. Furthermore, we provide the limiting distribution for the average lifetime of the entities in the system. In Section 4, we give an outlook on future research.

\section{Computations in the bivariate Freund $(a, 2)$ model}

\subsection{Tail dependence}

Definition 1. The lower and upper tail dependence coefficients for a bivariate copula are defined by

$$
\lambda_{L}=\lim _{u \rightarrow 0+} \frac{C(u, u)}{u}, \quad \lambda_{U}=\lim _{u \rightarrow 1-} \frac{1-2 u+C(u, u)}{1-u} .
$$

Proposition 1. The lower tail dependence coefficient for the bivariate Freund $(A)$ copula is

$$
\lambda_{L}= \begin{cases}0 & \text { for all } \lambda_{1}>0, \lambda_{2}>0, a_{1} \geq 0, a_{2} \geq 0, \min \left\{a_{1}, a_{2}\right\}<\infty \\ 1 & \text { for all } \lambda_{1}>0, \lambda_{2}>0, a_{1}=a_{2}=\infty\end{cases}
$$

Proof. If $\min \left\{a_{1}, a_{2}\right\}<\infty$, then using the first order Taylor expansion of $F(x)$ and $G(x)$ (given in (4) and (5)) around the basis point $x_{0}=0$, one can write $F(x)=\lambda_{1} \cdot x+O\left(x^{2}\right)$ and $G(x)=\lambda_{2} \cdot x+O\left(x^{2}\right)$ as $x \rightarrow$ $0+$. Therefore $F^{-1}(u)=\frac{1}{\lambda_{1}} \cdot u+O\left(u^{2}\right)$ and $G^{-1}(u)=\frac{1}{\lambda_{2}} \cdot u+O\left(u^{2}\right)$ as $u \rightarrow 0+$. Using (3), we can write $\lambda_{L}=\lim _{u \rightarrow 0+} \frac{C(u, u)}{u}=\lim _{u \rightarrow 0+} \frac{H\left(F^{-1}(u), G^{-1}(u)\right)}{u}=0$, where the indeterminate form appearing in the limit can be computed either by the first order Taylor expansion of the exponential function or by L'Hospital's rule. If $a_{1}=a_{2}=\infty$, the copula function is $C(u, v)=\min \{u, v\}$, so $\lambda_{L}=\lim _{u \rightarrow 0+}=\frac{C(u, u)}{u}=1$.

Remark 1. The first case of the statement $\left(\lambda_{L}=0\right)$ is a direct consequence of a much more general but simple fact. For the details see Appendix D. 
Proposition 2. The upper tail dependence coefficient for the bivariate Freund $(A)$ copula is given by

$$
\lambda_{U}=\left\{\begin{array}{cc}
1-\frac{\lambda_{1}}{a_{2}-\lambda_{1}} \cdot\left(1-\frac{\lambda_{2}}{a_{1}}\right)^{\frac{\lambda_{1}-a_{2}}{\lambda_{1}+\lambda_{2}}} \cdot\left(1-\frac{\lambda_{1}}{a_{2}}\right)^{\frac{\lambda_{2}+a_{2}}{\lambda_{1}+\lambda_{2}},} & \text { if } \begin{array}{c}
a_{1}>\lambda_{2}, a_{2}>\lambda_{1}, \\
a_{1} \cdot \lambda_{1} \geq \lambda_{2} \cdot a_{2},
\end{array} \\
1-\frac{\lambda_{2}}{a_{1}-\lambda_{2}} \cdot\left(1-\frac{\lambda_{1}}{a_{2}}\right)^{\frac{\lambda_{2}-a_{1}}{\lambda_{1}+\lambda_{2}}} \cdot\left(1-\frac{\lambda_{2}}{a_{1}}\right)^{\frac{\lambda_{1}+a_{1}}{\lambda_{1}+\lambda_{2}},}, & \text { if } \begin{array}{c}
a_{1}>\lambda_{2}, \\
a_{1} \cdot a_{1} \leq \lambda_{2} \cdot \lambda_{1},
\end{array}, \quad \text { (ii) } \\
0 & \text { otherwise (iii)-(viii) }
\end{array}\right.
$$

Proof. In addition to the indicated cases (i) and (ii), we distinguish the following cases as well.
(iii) $a_{1}<\lambda_{2}, a_{2}<\lambda_{1}$,
(iv) $a_{1}>\lambda_{2}, a_{2}<\lambda_{1}$,
(v) $a_{1}<\lambda_{2}, a_{2}>\lambda_{1}$,
(vi) $a_{1} \neq \lambda_{2}, a_{2}=\lambda_{1}$,
(vii) $a_{1}=\lambda_{2}, a_{2} \neq \lambda_{1}$,
(viii) $a_{1}=\lambda_{2}$ and $a_{2}=\lambda_{1}$.

The basic idea in each case is that one of the two exponential terms in formulas (4) and (5) of the marginal cdfs $F(x)$ and $G(x)$ is negligible if $x$ is large enough. This simplification enables us to give an approximation for $F^{-1}(u)$ and $G^{-1}(u)$ when $u$ is near to 1 . We present the proofs for (i), and (vi). The remaining cases are similar to these.

To see (i):

Under the above assumptions on parameters $\lambda_{1}, \lambda_{2}, a_{1}, a_{2}$, the approximation

$F(x)=1-\frac{a_{1} \cdot e^{-\left(\lambda_{1}+\lambda_{2}\right) x}}{a_{1}-\lambda_{2}}+O\left(e^{-\left(\lambda_{1}+a_{1}\right) x}\right)$ holds as $x \rightarrow \infty$,

therefore $F^{-1}(u)=-\frac{1}{\lambda_{1}+\lambda_{2}} \cdot \log \left(\left(1-\frac{\lambda_{2}}{a_{1}}\right) \cdot(1-u)\right)+O(\log u)$ as $u \rightarrow 1-$.

Similarly, $G(x)=1-\frac{a_{2} \cdot e^{-\left(\lambda_{1}+\lambda_{2}\right) x}}{a_{2}-\lambda_{1}}+O\left(e^{-\left(\lambda_{2}+a_{2}\right) x}\right)$ as $x \rightarrow \infty$,

so $G^{-1}(u)=-\frac{1}{\lambda_{1}+\lambda_{2}} \cdot \log \left(\left(1-\frac{\lambda_{1}}{a_{2}}\right) \cdot(1-u)\right)+O(\log u)$ as $u \rightarrow 1-$.

Using formulas (3), (4), and (5), we can write $\lambda_{U}=\lim _{u \rightarrow 1-} \frac{1-2 u+C(u, u)}{1-u}=$

$\lim _{u \rightarrow 1-} \frac{1-2 u+H\left(F^{-1}(u), G^{-1}(u)\right)}{1-u}=1-\frac{\lambda_{1}}{a_{2}-\lambda_{1}} \cdot\left(1-\frac{\lambda_{2}}{a_{1}}\right)^{\frac{\lambda_{1}-a_{2}}{\lambda_{1}+\lambda_{2}}} \cdot\left(1-\frac{\lambda_{1}}{a_{2}}\right)^{\frac{\lambda_{2}+a_{2}}{\lambda_{1}+\lambda_{2}}}$, as it was claimed.

To see (vi):

We can assume without loss of generality that $a_{1}>\lambda_{2}$. It is easy to think over that in this case $F^{-1}(u) \leq G^{-1}(u)$, if $u$ is close to 1 .

We will compute $\lambda_{U}$ in the following form.

$$
\lambda_{U}=\lim _{x \rightarrow \infty} \frac{1-2 G(x)-H\left(F^{-1}(G(x)), x\right)}{1-G(x)} .
$$

Using the negligibility principle explained in the beginning of the proof, we can write $F^{-1}(u)=\frac{-1}{\lambda_{1}+\lambda_{2}} \cdot \log \left(\frac{a_{1}-\lambda_{2}}{a_{1}}(1-u)\right)+O(\log u)$, if $u \rightarrow 1-$, and

$G(x)=1-\lambda_{1} \cdot x \cdot e^{-\left(\lambda_{1}+\lambda_{2}\right) x}+O\left(e^{-\left(\lambda_{1}+\lambda_{2}\right) x}\right)$ as $x \rightarrow \infty$ (see also formulas (8) and (9) in [6]), so $F^{-1}(G(x))=x-\frac{1}{\lambda_{1}+\lambda_{2}} \cdot \log \left(\frac{a_{1}-\lambda_{2}}{a_{1}} \cdot \lambda_{1} \cdot x\right)+O\left(x \cdot e^{-\left(\lambda_{1}+\lambda_{2}\right) x}\right)$ as $x \rightarrow \infty$.

Substituting this into (8), and performing elementary limit computations, $\lambda_{U}=0$ follows.

\subsection{The componentwise maxima of the lifetime variable $\underline{X} \sim \operatorname{Freund}(a, 2)$}

When one comes across a bivariate distribution and the corresponding bivariate copula, it is a natural question whether the distribution or the copula possesses some remarkable properties, for instance, whether they are max-stable or not. In order to investigate this, we introduce the componentwise maximum across $N$ independent copies $\left(X_{1}^{(i)}, X_{2}^{(i)}\right) \sim \operatorname{Freund}(a, 2),(i=1, \ldots, N)$, which will be denoted by

$$
M_{N}^{(j)}:=\max _{1 \leq i \leq N}\left\{X_{j}^{(i)}\right\} \text { for } j=1,2 .
$$

Since the law of $M_{N}^{(1)}$ coincides with that of $M_{N}^{(2)}$, we will omit the upper index from the notation, unless both quantities appear in the same context (e.g., in Subsection 2.3 and 2.4). In accordance with this, we will 
use the notation $X^{(i)}$ as well. In this subsection, we investigate the fundamental characteristics (the expectation $\mathbb{E}$ and the variance $\mathbb{V}$ ) of $M_{n}$, and we provide a non-trivial limiting distribution for it after suitable normalization. In Subsection 2.3, we examine the joint behavior of $M_{N}^{(1)}$ and $M_{N}^{(2)}$, including their copula $C_{N}^{(a)}$. In Subsection 2.4, we derive the limiting case of $C_{N}^{(a)}$ as $N \rightarrow \infty$, which, to the best of our knowledge, is a new extreme value copula. As a consequence, we will see that the Freund copula itself is not max-stable, except when $a=\infty$.

\subsubsection{Expectation and variance for $\boldsymbol{N}=\mathbf{2}$ and for large $\boldsymbol{N}$}

The cdf of $M_{N}$ is given by

$$
F_{N}^{\star}(x):=\mathbb{P}\left(M_{N} \leq x\right)=(F(x))^{N}, \quad x \geq 0,
$$

where $F$ is given by setting $\lambda_{1}=\lambda_{2}=1$ and $a_{1}=a$ in (4). In principle, it is easy to compute $\mathbb{E}\left(M_{N}\right), \mathbb{E}\left(M_{N}^{2}\right)$ and $\mathbb{D}\left(M_{N}\right)$, i.e., the mean, the second moment and the standard deviation, because all appearing integrals only consist of products of polynomials and exponential functions, where the degree of polynomials is zero, one, or two. However, in practice this task is much harder.

We performed these computations for $N=2$ and for comparison, we recall also the case $N=1$. For fixed $N>2$, we provide $\mathbb{E}\left(M_{N}\right)$ only in the extreme cases $a=0$ and $a=\infty$. For large $N$ we refer to the asymptotics that will be presented in Corollary 1 after Proposition 3 (see (18) and also Table 1). For $N=1$, we have $\mathbb{E}\left(M_{1}\right)=\mathbb{E}\left(X_{1}\right)=\frac{1}{2} \cdot \frac{a+2}{a+1}$ trivially, as it was shown in [6], Section 3.1., p.34. Notice that $\mathbb{E}\left(M_{1}\right)=1$ for $a=0$ and $\mathbb{E}\left(M_{1}\right)=\frac{1}{2}$ for $a=\infty$. For $N=2$, one gets by a cumbersome computation that

$$
\begin{gathered}
\mathbb{E}\left(M_{2}\right)=\frac{1}{4} \cdot \frac{3 a^{2}+14 a+18}{(a+1)(a+3)}, \\
\mathbb{E}\left(M_{2}^{2}\right)=\frac{7 a^{4}+62 a^{3}+207 a^{2}+344 a+252}{8(a+1)^{2}(a+3)^{2}}, \\
\mathbb{V}\left(M_{2}\right)=\frac{5 a^{4}+40 a^{3}+110 a^{2}+184 a+180}{16(a+1)^{2}(a+3)^{2}} .
\end{gathered}
$$

Notice that for $a=0$, formulas (10)-(12) yield $\mathbb{E}\left(M_{2}\right)=\frac{3}{2}, \mathbb{E}\left(M_{2}^{2}\right)=\frac{7}{2}$ and $\mathbb{V}\left(M_{2}\right)=\frac{5}{4}$, while for $a=\infty$, one gets $\mathbb{E}\left(M_{2}\right)=\frac{3}{4}, \mathbb{E}\left(M_{2}^{2}\right)=\frac{7}{8}$ and $\mathbb{V}\left(M_{2}\right)=\frac{5}{16}$.

For a general fixed $N$, we restrict our investigation to the extreme cases.

If $a=0$, then $X^{(i)}$ s are i.i.d. $\operatorname{Exp}(1)$ distributed, so one gets that the expectation of the variable $M_{N}=$ $\max \left(X^{(1)}, \ldots, X^{(N)}\right)$ is

$$
\mathbb{E}\left(M_{N}\right)=\int_{0}^{\infty}\left(1-\left(1-e^{-\chi}\right)^{N}\right) d x=\int_{0}^{1} \frac{1-u^{N}}{1-u} d u=\int_{0}^{1} \sum_{k=0}^{N-1} u^{k} d u=\sum_{k=1}^{N} \frac{1}{k} .
$$

If $a=\infty$, then $X^{(i)}$ s are i.i.d. $\operatorname{Exp}(2)$ distributed, so, taking also into account the extreme case $a=0$ and its result in (13), one gets that the expectation of the variable $M_{N}=\max \left(X^{(1)}, \ldots, X^{(N)}\right)$ is

$$
\mathbb{E}\left(M_{N}\right)=\frac{1}{2} \sum_{k=1}^{N} \frac{1}{k}
$$

An alternative way for deriving (13) and (14) can be found in Spivey [18].

\subsubsection{Limiting distribution}

Proposition 3. The marginal cdfs $F$ and $G$ of the Freund $(a, 2)$ distribution belong to the domain of attraction of the standard Gumbel distribution. Namely,

$$
\lim _{N \rightarrow \infty} \mathbb{P}\left(\frac{M_{N}-b_{N}}{a_{N}} \leq x\right)=e^{-e^{-x}}
$$


where the normalizing constants are

$$
\begin{gathered}
a_{N}=\frac{1}{1+a}, \quad b_{N}=\frac{1}{1+a} \cdot \log \left(\frac{N}{1-a}\right), \quad \text { if } 0 \leq a<1, \\
a_{N}=\frac{1}{2}, \quad b_{N}=\frac{1}{2} \cdot \log \left(\frac{a \cdot N}{a-1}\right), \quad \text { if } a>1 . \\
a_{N}=\frac{1}{2}, \quad b_{N}=\frac{1}{2} \cdot \log N, \quad \text { if } \quad a=1 .
\end{gathered}
$$

Proof. We will use the sufficient condition on a $\operatorname{cdf} F$ for belonging to the domain of attraction of the Gumbel distribution stated for instance in Exercise 3.2 in R. Sun [19], and based on the classical works Leadbetter et al. [9] and Resnick [16].

For $a \neq 1$, we introduce the function $h(x)=-\log \left(\frac{1}{1-a} \cdot e^{-(1+a) \cdot x}-\frac{a}{1-a} \cdot e^{-2 x}\right)$. The marginal distribution function $F(x)$ can be written as $F(x)=1-e^{-h(x)}$. It is easy to see that $h^{\prime}(x)$ is a slowly varying function. This means that condition (i) in Exercise 3.2 in [19] holds, therefore $F(x)$ belongs to the domain of attraction of the Gumbel distribution.

For $a=1$, we introduce the function $h(x)=-\log (1+x)+2 x$. The marginal distribution function $F(x)$ can be written as $F(x)=1-e^{-h(x)}$ (see (6)). It is easy to see that $h(x)$ is of the form $h(x)=x \cdot L(x)$, where $L(x)$ is a slowly varying function; therefore, condition (ii) in Exercise 3.2 in [19] holds, and we obtain that $F(x)$ belongs to the domain of attraction of the Gumbel distribution in this case as well.

The normalizing constants $a_{N}, b_{N}$ for $a \neq 1$ can be computed analogously to Example 3.3 in [19], and by elementary considerations for $a=1$.

Remark 2. The general theoretical background of the normalizing constants can be found for instance in Resnick [16].

Corollary 1. The expectation of $M_{N}$ is essentially proportional to $\log (N)$ for large $N$. The precise statement can be formulated as

$$
\begin{gathered}
\lim _{N \rightarrow \infty}\left(\mathbb{E}\left(M_{N}\right)-\frac{1}{1+a} \cdot \log \left(\frac{N}{1-a}\right)\right)=\frac{\gamma}{1+a}, \quad \text { if } 0 \leq a<1, \\
\lim _{N \rightarrow \infty}\left(\mathbb{E}\left(M_{N}\right)-\frac{1}{2} \cdot \log (N)\right)=\frac{1}{2} \cdot \gamma \text { if } a=1, \\
\lim _{N \rightarrow \infty}\left(\mathbb{E}\left(M_{N}\right)-\frac{1}{2} \cdot \log \left(\frac{a \cdot N}{a-1}\right)\right)=\frac{1}{2} \cdot \gamma \quad \text { if } a>1,
\end{gathered}
$$

where $\gamma \approx 0.5772$ is the Euler-Mascheroni constant.

Definition 2. The normalized componentwise maximum is defined as $\widetilde{M}_{N}=\frac{M_{N}-b_{N}}{a_{N}}$, where $a_{N}$ and $b_{N}$ are given in (15)-(17). (See also Figure 1.)

Notice in Figure 1 that the convergence of $\widetilde{M}_{N}$ to its limiting distribution is very fast. The histograms for $N=10,100,1000$ are visually hardly distinguishable.

\subsection{Joint distribution and correlation between the componentwise maxima}

In the following, we will examine the relation between the componentwise maxima $M_{N}^{(1)}$ and $M_{N}^{(2)}$. Their joint cdf can be easily determined:

$$
H^{\star}(x, y):=\mathbb{P}\left(M_{N}^{(1)} \leq x, M_{N}^{(2)} \leq y\right)=(H(x, y))^{N}, \quad x, y \geq 0,
$$


Table 1: Expectation of the componentwise maximum in $N$ independent copies of Freund $(a, 2)$.

\begin{tabular}{|c|c|c|c|}
\hline$N$ & $\mathbb{E}\left(M_{N}\right)$ & $\mathbb{E}\left(M_{N}\right)$ for $a=0$ & $\mathbb{E}\left(M_{N}\right)$ for $a=\infty$ \\
\hline 1 & $\frac{1}{2} \cdot \frac{a+2}{a+1}$ & 1 & $\frac{1}{2}$ \\
\hline 2 & $\frac{1}{4} \cdot \frac{3 a^{2}+14 a+18}{(a+1)(a+3)}$ & $\frac{3}{2}$ & $\frac{3}{4}$ \\
\hline general $N$ & a rational function of $a$ & $\sum_{k=1}^{N} \frac{1}{k}$ & $\frac{1}{2} \cdot \sum_{k=1}^{N} \frac{1}{k}$ \\
\hline$N \gg 1$ & see (18) for the order of $\mathbb{E}\left(M_{N}\right)$ & $\sum_{k=1}^{N} \frac{1}{k} \approx \gamma+\log N$ & $\frac{1}{2} \cdot \sum_{k=1}^{N} \frac{1}{k} \approx \frac{1}{2} \cdot(\gamma+\log N)$ \\
\hline
\end{tabular}
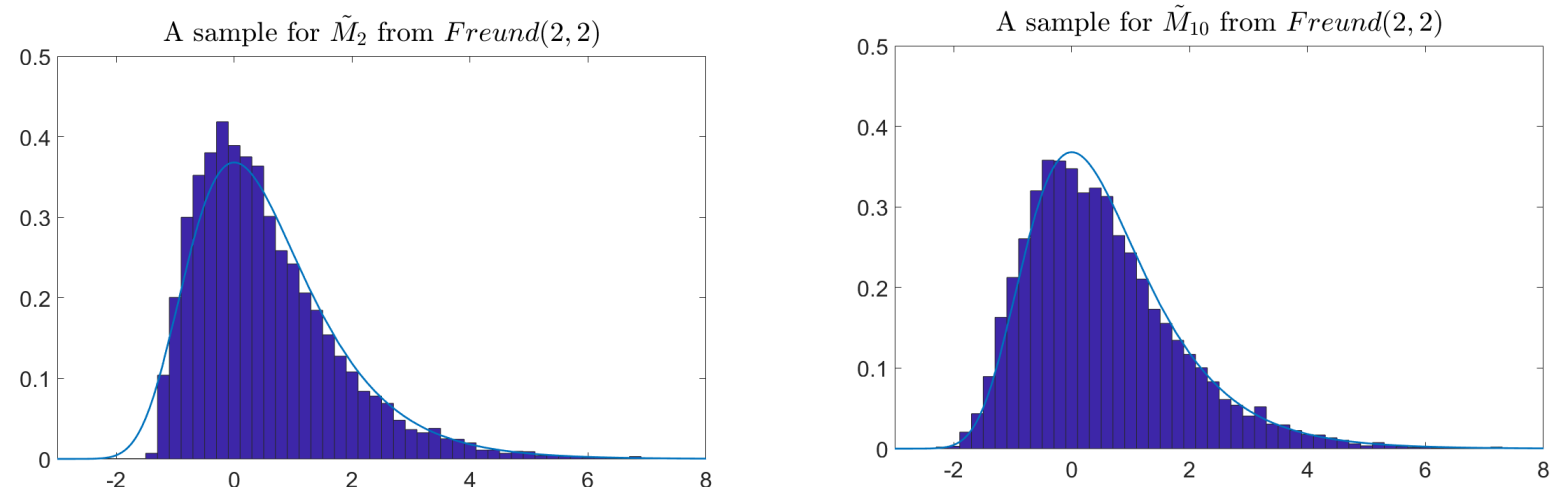

A sample for $\tilde{M}_{100}$ from Freund $(2,2)$
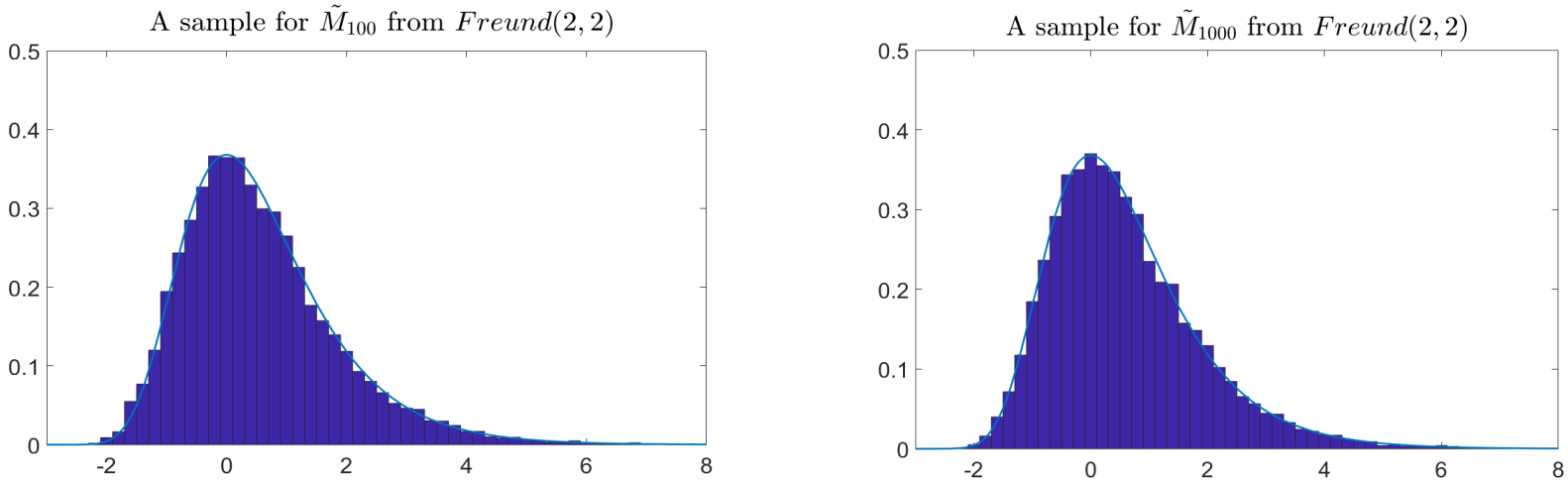

Figure 1: The limiting distribution of the suitably normalized componentwise maximum is the standard Gumbel distribution. The figure illustrates the convergence by samples of size 10000 .

where $H(x, y)$ is the cumulative distribution function of the $\operatorname{Freund}(a, 2)$ distribution. Therefore $\operatorname{corr}\left(M_{N}^{(1)}, M_{N}^{(2)}\right)$ can be also determined, since all the computations consist of integrals of functions comprising a polynomial times exponential, where the polynomial has degree zero, one, or two. We performed the computation for $N=2$.

For the cross-product we have found that

$$
\mathbb{E}\left(M_{2}^{(1)} \cdot M_{2}^{(2)}\right)=\frac{7 a^{4}+62 a^{3}+199 a^{2}+290 a+162}{8(a+1)^{2}(a+3)^{2}},
$$


and using formulas (10)-(12), one gets

$$
\operatorname{corr}\left(M_{2}^{(1)}, M_{2}^{(2)}\right)=\frac{5 a^{4}+40 a^{3}+94 a^{2}+76 a}{5 a^{4}+40 a^{3}+110 a^{2}+184 a+180} .
$$

From (19), we immediately see that $\operatorname{corr}\left(M_{2}^{(1)}, M_{2}^{(2)}\right)$ is increasing in $a$. The two extreme cases are $\operatorname{corr}\left(M_{2}^{(1)}, M_{2}^{(2)}\right)=0$ for $a=0$ and $\operatorname{corr}\left(M_{2}^{(1)}, M_{2}^{(2)}\right)=1$ for $a=\infty$. It also means that $M_{2}^{(1)}$ and $M_{2}^{(2)}$ are not independent if $a>0$. Figure $2 \mathrm{~b}$ shows a sample for $a=2$; the theoretical correlation is $\operatorname{corr}\left(M_{2}^{(1)}, M_{2}^{(2)}\right)=$ $\frac{232}{347} \simeq 0.6686$.

\subsection{The limiting case of the copula of the componentwise maxima: a new extreme-value copula}

It is worth examining how the dependence between $M_{N}^{(1)}$ and $M_{N}^{(2)}$ changes as $N$ varies. (See also Figure 2 and Figure 3.) Although the scatterplots of the copula of $M_{N}^{(1)}$ and $M_{N}^{(2)}$ for $a=2$ do not seem to significantly differ from the copula of $\left(X_{1}, X_{2}\right)$, we will see that the distribution of $\left(X_{1}, X_{2}\right)$ is not max-stable provided that $a<\infty$. In Proposition 4, we will derive an analytic formula for the extreme-value copula which stems from $\left(X_{1}, X_{2}\right)$. Two cases need to be distinguished: $0 \leq a \leq 1$, and $a>1$. The latter case, we get an extreme value copula which, to the best of our knowledge, has not been discussed in the literature yet, so in fact we have found a new copula (what is more, an extreme value copula) that can be given explicitly.

Before presenting this computation, we numerically quantify the phenomenon pictured in Figure 2, which visually suggests that the copulas of $\left(M_{N}^{(1)}, M_{N}^{(2)}\right)$ are "similar" to each other practically for all values of $N$. We want to express more exactly whether these copulas are indeed close to each other. The second and fourth columns of Table 2 show the sup-distance and the mean-absolute-distance between the copula of $\left(X_{1}, X_{2}\right)$ (denoted by $C_{1}$ in Table 2) and the copula of $\left(M_{N}^{(1)}, M_{N}^{(2)}\right)$ (denoted by $C_{N}$ in Table 2). The third and fifth columns of the table use the notation $C_{N_{i-1}}$ and $C_{N_{i}}$, referring to the $N$ values which appear in the $(i-1)$-th and $i$-th row of the table, i.e., always in the current and in the previous row.

Table 2: Distances of copulas with all copulas stemming from the Freund(2, 2) model.

\begin{tabular}{|c||c|c|c|c|}
\hline$N$ & $\begin{array}{c}\text { sup distance } \\
\text { of } C_{1} \text { and } C_{N}\end{array}$ & $\begin{array}{c}\text { sup distance } \\
\text { of } C_{N_{i-1}} \text { and } C_{N_{i}}\end{array}$ & $\begin{array}{c}\text { mean abs distance } \\
\text { of } C_{1} \text { and } C_{N}\end{array}$ & $\begin{array}{c}\text { mean abs distance } \\
\text { of } C_{N_{i-1}} \text { and } C_{N_{i}}\end{array}$ \\
\hline \hline 2 & 0.031 & $(0.031)$ & 0.00579 & $(0.00579)$ \\
\hline 5 & 0.0039 & 0.018 & 0.00774 & 0.00370 \\
\hline 10 & 0.039 & 0.019 & 0.00841 & 0.00437 \\
\hline 100 & 0.033 & 0.024 & 0.00711 & 0.00500 \\
\hline 1000 & 0.030 & 0.021 & 0.00662 & 0.00350 \\
\hline 10000 & 0.029 & 0.024 & 0.00536 & 0.00480 \\
\hline
\end{tabular}

The computations in Table 2 are based on samples of size 1000 for $\left(X_{1}, X_{2}\right)$ and $\left(M_{N}^{(1)}, M_{N}^{(2)}\right)$. The copula function values are approximated by the empirical copula function values. The empirical copulas (based on the above mentioned samples) were evaluated at the points $(i / 100, j / 100), i, j=1, \ldots, 100$. A larger sample size and a finer grid might lead to slightly different numerical values. 
Empirical copula of $\left(X_{1}, X_{2}\right)$ from Freund $(2,2)$

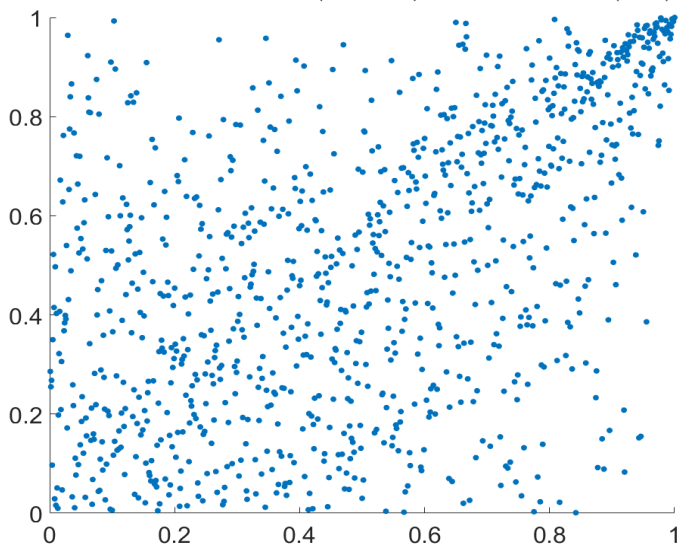

(a) $\operatorname{corr}\left(X_{1}, X_{2}\right)=\frac{a \cdot(a+2)}{(a+1)^{2}+3}=\frac{2}{3}(0.6710$ in the sample).

Empirical copula of $\left(M_{10}^{(1)}, M_{10}^{(2)}\right)$ from Freund $(2,2)$

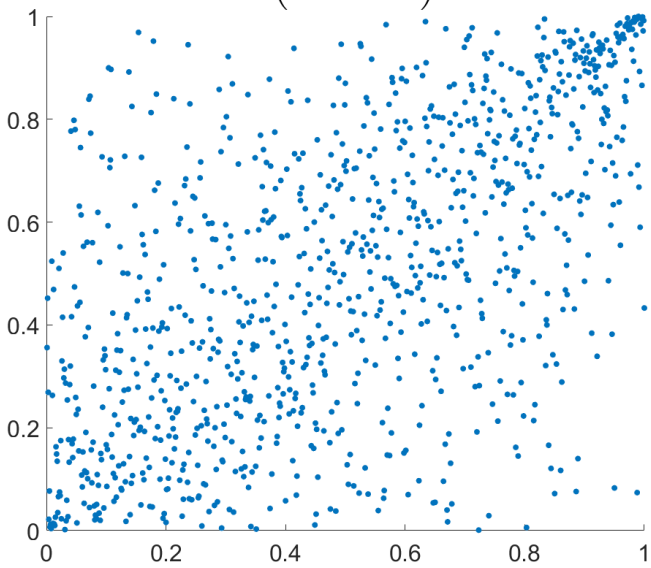

(c) $\operatorname{corr}\left(M_{10}^{(1)}, M_{10}^{(2)}\right) \simeq 0.6311$ in the current sample.
Empirical copula of $\left(M_{2}^{(1)}, M_{2}^{(2)}\right)$ from $\operatorname{Freund}(2,2)$

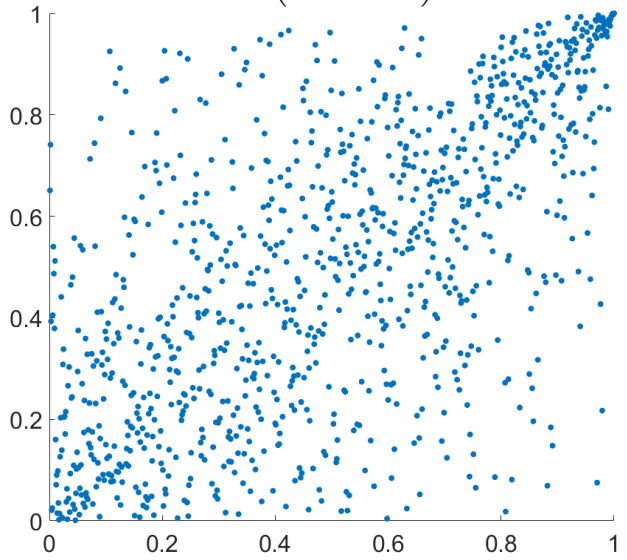

(b) $\operatorname{corr}\left(M_{2}^{(1)}, M_{2}^{(2)}\right) \simeq 0.7163$ in the current sample.

Empirical copula of $\left(M_{10000}^{(1)}, M_{10000}^{(2)}\right)$ from Freund $(2,2)$

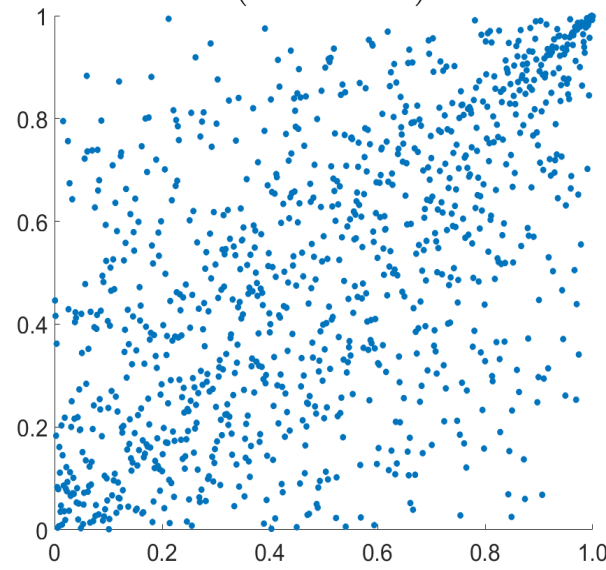

(d) $\operatorname{corr}\left(M_{10000}^{(1)}, M_{10000}^{(2)}\right) \simeq 0.6731$ in the current sample.

Figure 2: Scatterplots of componentwise maxima for $a=2$.

Proposition 4. Let $C_{N}^{(a)}$ denote the copula of $M_{N}^{(1)}$ and $M_{N}^{(2)}$ taken from the underlying model Freund $(a, 2)$.

(i) If $0 \leq a \leq 1$, then

$$
\widetilde{C}^{(a)}(u, v):=\lim _{N \rightarrow \infty} C_{N}^{(a)}(u, v)=\Pi(u, v)=u \cdot v \quad \text { for } 0 \leq u, v \leq 1
$$

(ii) If $a>1$, then

$$
\widetilde{C}^{(a)}(u, v)=\lim _{N \rightarrow \infty} C_{N}^{(a)}(u, v)=\left\{\begin{array}{cl}
u \cdot e^{-\frac{1}{a} \cdot\left((-\log u)^{\frac{1-a}{2}} \cdot(-\log v)^{\frac{1+a}{2}}\right)} & \text { if } 0<u \leq v \leq 1, \\
v \cdot e^{-\frac{1}{a} \cdot\left((-\log u)^{\frac{1+a}{2}} \cdot(-\log v)^{\frac{1-a}{2}}\right)} & \text { if } 0<v \leq u \leq 1, \\
0 & \text { if } u=0 \text { or } v=0 .
\end{array}\right.
$$

Proof. It is well-known, and one can also verify by trivial considerations, that between a bivariate copula $C(u, v)$ and the corresponding copula $C_{N}(u, v)$ of the componentwise maxima, the following relation holds:

$$
C_{N}(u, v)=C\left(u^{1 / N}, v^{1 / N}\right)^{N} .
$$


Our aim is to compute

$$
\widetilde{C}(u, v):=\lim _{N \rightarrow \infty} C_{N}(u, v)
$$

for the above defined $C_{N}^{(a)}$.

We will use a characterization which can be found (among others) in Gudendorf and Segers [5] and in Drees and Huang [3], by which the relation between $C$ and $\widetilde{C}$ can be written in terms of the the tail dependence function $\ell$ as follows:

$$
\ell(x, y)=-\log \widetilde{C}\left(e^{-x}, e^{-y}\right)=\lim _{t \rightarrow 0+} \frac{1-C(1-t \cdot x, 1-t \cdot y)}{t} .
$$

Applying this to the Freund ( $a, 2)$ model and also using the semi-explicit expression (7), one can write

$$
\ell(x, y)=\lim _{t \rightarrow 0+} \frac{1-H\left(F^{-1}(1-t x), G^{-1}(1-t y)\right)}{t} .
$$

In the remaining part of the proof, we resort to approximating the marginal cdfs and the inverse marginal cdfs in order to compute the limit in (23). The validity of these approximations can be verified in an elementary way.

To show (i) for $0 \leq a<1$, we need the following considerations.

When $x \rightarrow \infty$, then according to (4), the marginal cdf $F$ can be approximated as

$$
F(x)=1-\frac{1}{1-a} \cdot e^{-(1+a) x}+O\left(e^{-2 x}\right)
$$

Therefore, $F^{-1}(u)$ can be approximated as

$$
F^{-1}(u)=-\frac{1}{1+a} \cdot \log ((1-a)(1-u))+O(\log u), \text { as } u \rightarrow 1-.
$$

As a consequence, the approximation

$$
F^{-1}(1-t x)=-\frac{\log ((1-a) t x)}{1+a}+O(\log (1-t x))=-\frac{\log ((1-a) t x)}{1+a}+o(1) \text { as } t \rightarrow 0+
$$

can be used when we compute the limit in (23). Analogously, the approximation

$$
G^{-1}(1-t y)=-\frac{\log ((1-a) t y)}{1+a}+o(1) \text { as } t \rightarrow 0+
$$

holds for the marginal $\operatorname{cdf} G$.

Substituting (26) and (27) into (23) and also using (3), one gets after taking the limit

$$
\ell(x, y)=x+y
$$

which yields

$$
\widetilde{C}^{(a)}(u, v)=e^{-\ell(-\log u,-\log v)}=u \cdot v,
$$

as it was claimed.

The proof of (i) for $a=1$ is similar to the case $0 \leq a<1$. The only difference is that one has to use the following approximations based on (6):

$$
\begin{aligned}
& F(x)=1-x e^{-2 x}+O\left(e^{-2 x}\right) \quad \text { as } x \rightarrow \infty, \\
& G(y)=1-y e^{-2 y}+O\left(e^{-2 y}\right) \quad \text { as } y \rightarrow \infty,
\end{aligned}
$$

and therefore

$$
\begin{aligned}
& F^{-1}(u)=-\frac{1}{2} \cdot \log (1-u)+O(\log u) \text { as } u \rightarrow 1-, \\
& G^{-1}(v)=-\frac{1}{2} \cdot \log (1-v)+O(\log v) \text { as } v \rightarrow 1-
\end{aligned}
$$


To show (ii), first we assume that $0<u \leq v$.

When $x \rightarrow \infty, y \rightarrow \infty$, then (according to (4) and (5)) $F(x)=1-\frac{a}{a-1} \cdot e^{-2 x}+O\left(e^{-(1+a) x}\right)$ and $G(y)=$ $1-\frac{a}{a-1} \cdot e^{-2 y}+O\left(e^{-(1+a) y}\right)$, and therefore

$$
\begin{gathered}
F^{-1}(u)=-\frac{1}{2} \cdot \log \left(\frac{a-1}{a} \cdot(1-u)\right)+O(\log u), \text { as } u \rightarrow 1-, \text { and } \\
F^{-1}(1-t \cdot x)=-\frac{1}{2} \log \left(\frac{a-1}{a} \cdot t \cdot x\right)+O(\log (1-t x))=-\frac{1}{2} \log \left(\frac{a-1}{a} \cdot t \cdot x\right)+o(1) \text { as } t \rightarrow 0+.
\end{gathered}
$$

Similarly, $G^{-1}(v)=-\frac{1}{2} \cdot \log \left(\frac{a-1}{a} \cdot(1-v)\right)+O(\log v)$, as $v \rightarrow 1-$, and

$$
G^{-1}(1-t \cdot y)=-\frac{1}{2} \log \left(\frac{a-1}{a} \cdot t \cdot y\right)+O(\log (1-t y))=-\frac{1}{2} \log \left(\frac{a-1}{a} \cdot t \cdot y\right)+o(1) \text { as } t \rightarrow 0+.
$$

Substituting (28) and (29) into (23) and also using (3), one gets after taking the limit

$$
\begin{gathered}
\ell(x, y)=x+\frac{1}{a} \cdot x^{\frac{1-a}{2}} \cdot y^{\frac{1+a}{2}}, \quad \text { which yields } \\
\widetilde{C}^{(a)}(u, v)=e^{-\ell(-\log u,-\log v)}=u \cdot e^{-\frac{1}{a} \cdot\left((-\log u)^{\frac{1-a}{2}} \cdot(-\log v)^{\frac{1+a}{2}}\right)}, \quad \text { as it was claimed. }
\end{gathered}
$$

Showing (ii) for the case $0<v \leq u$ is similar and for the case $u=0$ or $v=0$ is trivial.

Figure 3 illustrates Proposition 4 (i) for $a=0.99$. One can immediately see that the convergence to the independence copula $\Pi$ is relatively slow; however this effect is hard to observe visually based on the scatterplots. (It is also obvious that smaller values of $a$ would lead to faster convergence, since the original variables $\left(X_{1}, X_{2}\right)$ are more independent and in case $a=0$ actually independent.)

Remark 3. The copula $\widetilde{C}^{(a)}(u, v)$ is continuous in the parameter $a$. This is trivial for all $a \neq 1$ and can be verified for $a=1$ by evaluating (21) for $a=1$.

Corollary 2. The result clearly shows that the Freund copula $C^{(a)}(u, v)$ for $0 \leq a<\infty$ is not max-stable, since for the max-stability $C^{(a)}(u, v)=\widetilde{C}^{(a)}(u, v)$ should hold, which is not the case.

Remark 4. According to Mathieu and Mohammed [13] and de Haan and Resnick [1] (bivariate) extreme value copulas can be also characterized by an exponent function $V$ such that

$$
\widetilde{C}(u, v)=e^{-V\left(-\frac{1}{\log u},-\frac{1}{\log v}\right)},
$$

where $V$ is a homogeneous function of order -1 . Looking at (21), it is easy to see that the exponent function $V$ corresponding to the $\operatorname{Freund}(a, 2)$ model (for $x \leq y$ ) is given by

$$
V(x, y)=\frac{1}{x}+\frac{1}{a} \cdot\left(x^{-\frac{1-a}{2}} \cdot y^{-\frac{1+a}{2}}\right)
$$

Proposition 5. Let $\widetilde{c}^{(a)}$ denote the copula density belonging to the extreme value copula $\widetilde{C}^{(a)}$ given in (20) and (21), i.e.,

$$
\widetilde{c}^{(a)}(u, v)=\frac{\partial^{2} \widetilde{C}^{(a)}(u, v)}{\partial u \partial v}
$$

(i) If $0 \leq a \leq 1$, then

$$
\widetilde{c}^{(a)}(u, v)=1 \text { for } 0 \leq u, v \leq 1
$$

(ii) If $a>1$, then

$$
\widetilde{c}^{(a)}(u, v)=
$$


Empirical copula of $\left(X_{1}, X_{2}\right)$ from Freund $(0.99,2)$

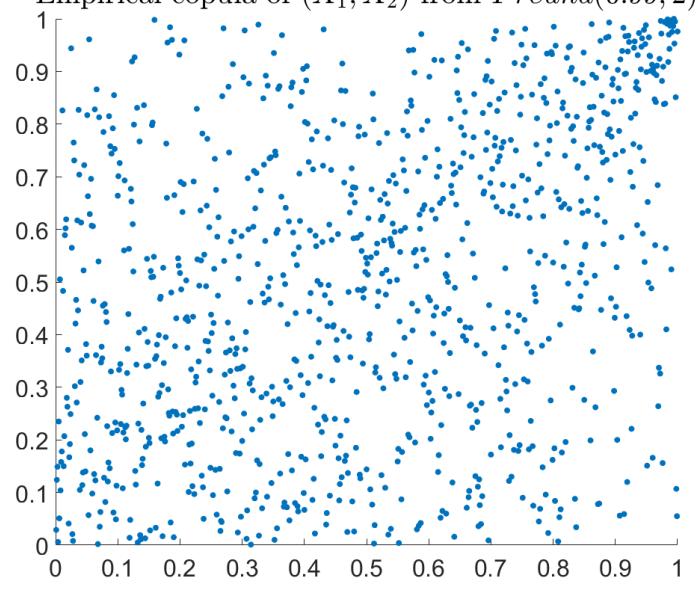

Empirical copula of $\left(M_{2}^{(1)}, M_{2}^{(2)}\right)$ from $\operatorname{Freund}(0.99,2)$

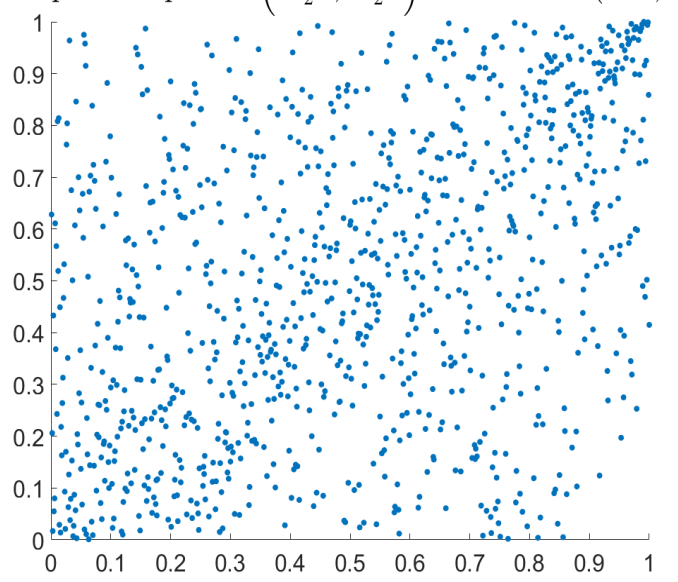

(a) $\operatorname{corr}\left(X_{1}, X_{2}\right)=\frac{a \cdot(a+2)}{(a+1)^{2}+3} \simeq 0.4253$ (0.4627 in the sample). (b) $\operatorname{corr}\left(M_{2}^{(1)}, M_{2}^{(2)}\right) \simeq 0.4471$.

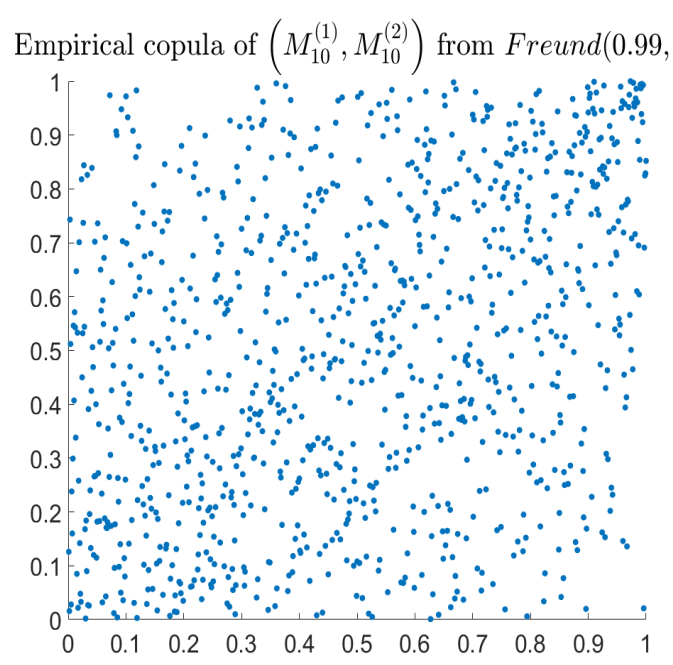

(c) $\operatorname{corr}\left(M_{10}^{(1)}, M_{10}^{(2)}\right) \simeq 0.3761$.
Empirical copula of $\left(M_{10000}^{(1)}, M_{10000}^{(2)}\right)$ from $\operatorname{Freund}(0.99,2)$

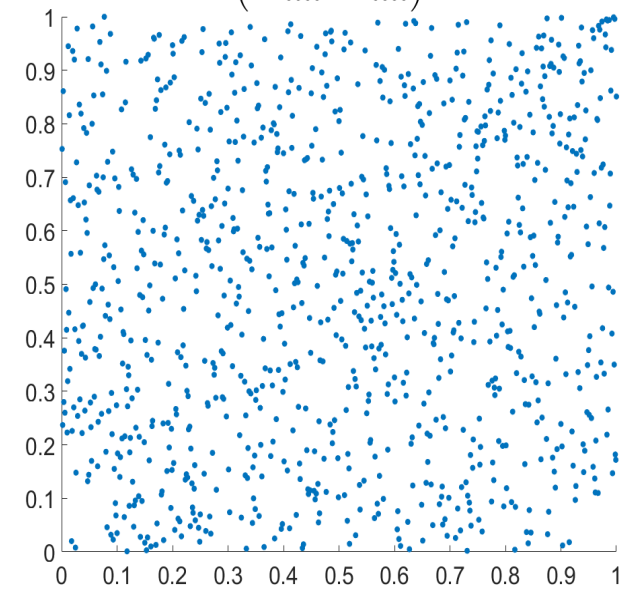

(d) $\operatorname{corr}\left(M_{10000}^{(1)}, M_{10000}^{(2)}\right) \simeq 0.1689$.

Figure 3: Scatterplots of componentwise maxima for $a=0.99$.

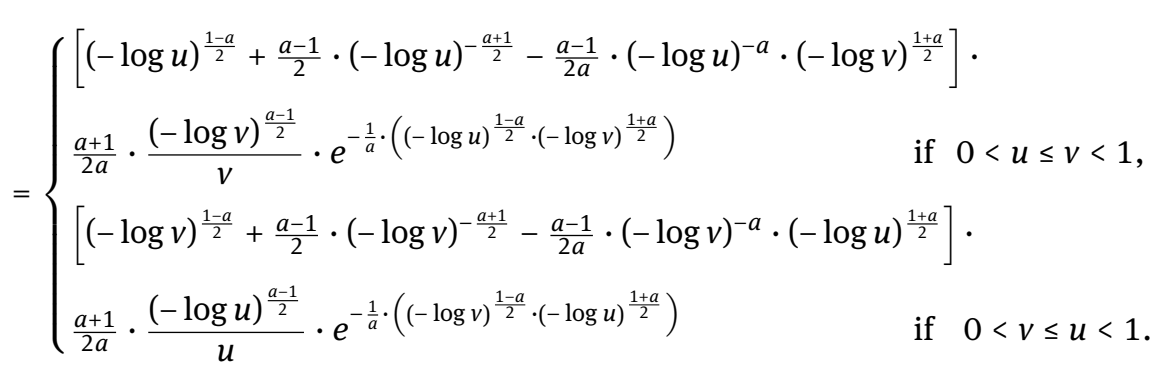

Proof. By simple differentiation.

Remark 5. For $a>1$, the copula density $\widetilde{c}^{(a)}$ is unbounded at $(0,0)$ and at $(1,1)$. One can see this by computing the suitable limits. The copula density is pictured in Figure 4 for $a=2$. 
The copula density $\tilde{c}^{(a)}(u, v)$ for $a=2$

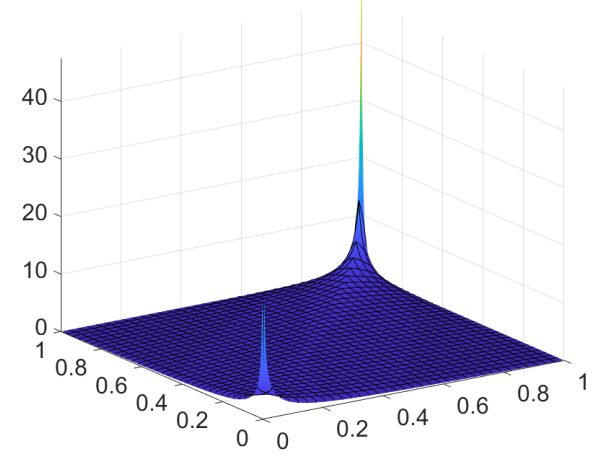

Figure 4

\subsection{Sampling from the new extreme value copula}

We present two methods for sampling from the new extreme value copula (21). According to our experiences, numerically none of them is significantly overperforming the other one. Figure 5 illustrates two samples obtained by these methods.

\subsubsection{Sampling by acceptance-rejection}

The idea of the acceptance-rejection method(s) (to the best of our knowledge) appeared first in von Neumann [21] and it also can be found in Chaper II.3 in Devroye [2], as a classical reference.

In order to find a suitable dominating density, one has to assure that both peaks of $c^{(a)}(u, v)$ (i.e., at around $(0,0)$ and $(1,1))$ are dominated. To this end, we will use the bivariate Clayton copula, a well-know copula family in the class of Archimedean copulas:

$$
C_{\vartheta}(u, v)=\left[\max \left\{u^{-\vartheta}+v^{-\vartheta}-1 ; 0\right\}\right]^{-1 / \vartheta}, \quad \vartheta \in[-1, \infty) \backslash\{0\} .
$$

The flipped Clayton copula can be found in many sources as well. As Hochrainer-Stigler et al. [7] mentions expressively: a (bivariate) flipped copula means that the copula is rotated by 180 degrees, and they also refer to Jongman et al. [8], where the formula of the flipped Clayton copula is given by

$$
\hat{C}_{\vartheta}(u, v)=u+v-1+\left[(1-u)^{-9}+(1-v)^{-\vartheta}-1\right]^{-1 / \vartheta}, \quad \vartheta \in[-1, \infty) \backslash\{0\} .
$$

Let us define the bivariate random vector $B=\left(B_{1}, B_{2}\right)$ as

$$
B:= \begin{cases}\mathcal{C}_{1} & \text { with probability } w, \\ \mathcal{C}_{2} & \text { with probability } w, \\ \mathcal{E} & \text { with probability } 1-2 w,\end{cases}
$$

where $\mathcal{C}_{1} \sim$ Clayton $(\vartheta), \mathcal{C}_{2} \sim$ flipped_Clayton $(\vartheta), \mathcal{E} \sim \Pi$ are independent of each other and the mixing parameter $w$ is between 0 and 0.5. ( $\Pi$ denotes the product copula, see for instance Proposition 4.) It is clear that the components $B_{1}$ and $B_{2}$ are Uni $[0,1]$ distributed, and therefore the joint density of the random vector $B$ is a copula density, which is denoted by $c_{B}$.

For the applicability of the acceptance-rejection method, we have to find a $K$ such that

$$
K \geq \max _{0<u, v<1} \frac{\widetilde{c}^{(a)}(u, v)}{c_{B}(u, v)},
$$


where $\widetilde{c}^{(a)}(u, v)$ is given in (31). Then one can draw a sample from the distribution of the random vector $B$, for instance by the well-known algorithm of Marshall and Olkin [12]. Finally, according to the classical acceptance-rejection method mentioned above, we gain a sample for the copula density $\widetilde{c}^{(a)}(u, v)$. Figure 5a shows such a sample, where the underlying distribution is a Freund $(2,2)$ distribution (i.e., $a=2$ ), the parameters are set to $\vartheta=60$ and $w=0.45$. We have found that the numerical solution of the maximization problem on the right-hand-side of (32) is about 18.18, and for the actual sampling we have chosen $K=25$.

\subsubsection{Sampling by numerically computing the quantiles of the conditional copula function}

The inverse conditional distribution method is well know (see for instance Nelsen [14], Section 2.9, or Mai and Scherer [10], Section 1.1.3). Suppose that the copula density is $c(u, v)$, which does not vanish in $[0,1]^{2}$, and let

$$
C_{v}(u):=\mathbb{P}(U \leq u \mid V=v)=\int_{0}^{u} c(w, v) d w=\frac{\partial C(u, v)}{\partial v} .
$$

Then the algorithm using Newton's method is as follows. Sample $V \sim \operatorname{Uni}[0,1]$ and independently $Z \sim$ Uni $[0,1]$. Set $u_{0}=0.5$ and consider the iteration

$$
u_{n+1}:=u_{n}+\frac{Z-C_{V}\left(u_{n}\right)}{c\left(u_{n}, V\right)} .
$$

Set $U=\lim _{n \rightarrow \infty} u_{n}$. The pair $(U, V)$ has the required copula density $c(u, v)$. For the practical implementation we used the stopping criterion $\left|u_{n}-u_{n-1}\right|<10^{-3}$.

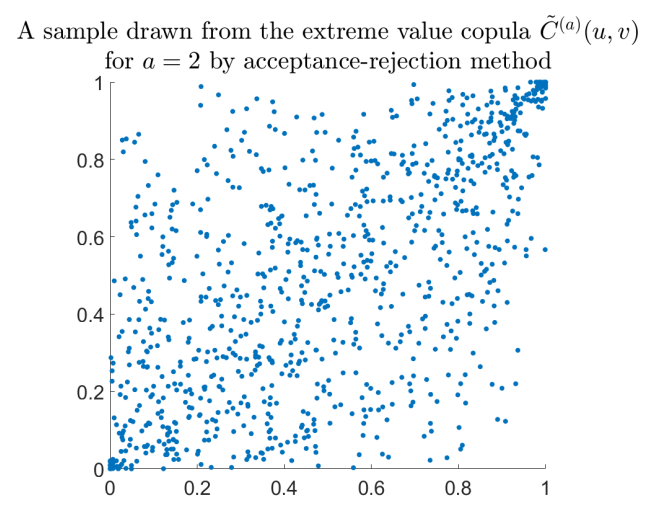

(a)

Figure 5: Two samples of size 1000 drawn from $\widetilde{C}^{(a)}(u, v)$ by two different methods.

(b)

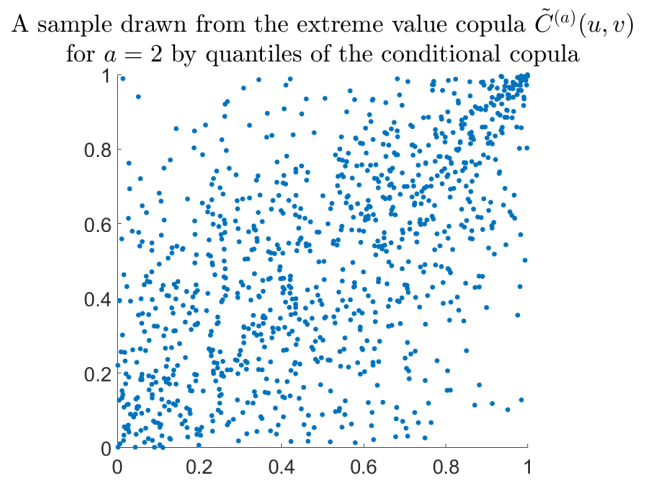

\subsection{Correlation coefficients (measures of concordance), Pickands dependence function and tail dependence coefficients of the new extreme value copula}

In this subsection, we investigate some charateristics of the newly explored extreme value copula. First we recall the definition of three usual correlation coefficients (measures of concordance), then we picture their values for $\tilde{C}^{(a)}(u, v)$ as functions of $a$. Second, we compute and picture Pickands dependence function, which is another usual way to characterize bivariate extreme value copulas. Third, we provide the tail dependence coefficients in Proposition 8. 
Definition 3. For a bivariate copula $C(u, v)$

(i) Spearman's $\rho$ is given by

$$
\rho=12 \cdot \int_{0}^{1} \int_{0}^{1} u v \mathrm{~d} C(u, v)-3,
$$

(ii) Kendall's $\tau$ is given by

$$
\tau=4 \cdot \int_{0}^{1} \int_{0}^{1} C(u, v) \mathrm{d} C(u, v)-1,
$$

(iii) Blomqvist's $\beta$ is given by

$$
\beta=4 \cdot C\left(\frac{1}{2}, \frac{1}{2}\right)-1
$$

Notice that in the trivial case $0 \leq a \leq 1$, all the three correlation coefficients are zero for $\tilde{C}^{(a)}(u, v)$.

Proposition 6. Let $a \geq 1$. Blomqvist's $\beta$ for the extreme value copula $\tilde{C}^{(a)}(u, v)$ is given by

$$
\beta=2^{\frac{a-1}{a}}-1 .
$$

Proof. Straightforward evaluation of (33) using (21).

When we aim to determine Spearman's $\rho$ and Kendall's $\tau$ for the extreme value copula $\tilde{C}^{(a)}(u, v)$, we cannot hope for analytic expressions. We performed these computations by numerical integration; the results, along with Blomqvist's $\beta$, are shown in Figure 6.

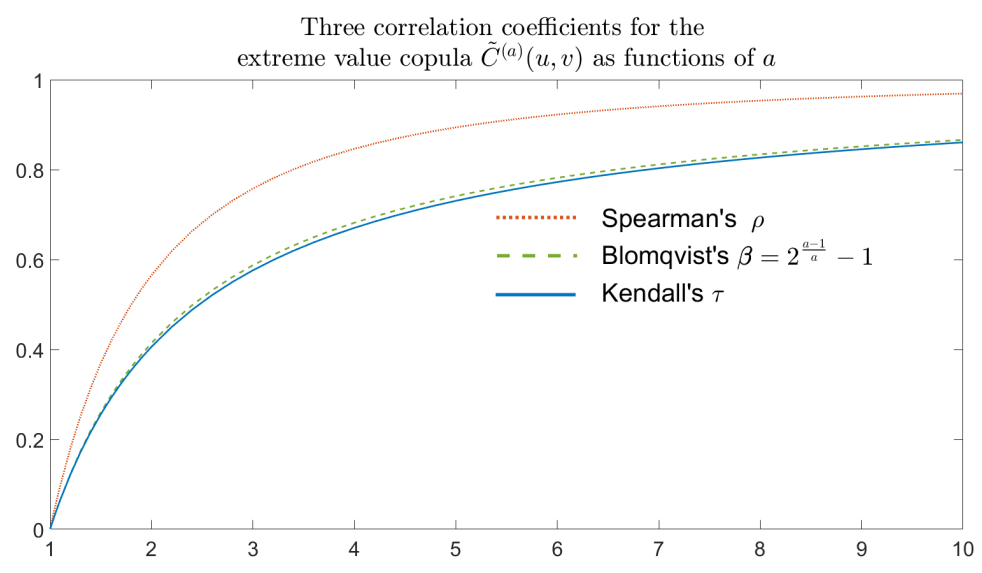

Figure 6

Definition 4. For a bivariate extreme value copula $\tilde{C}(u, v)$, Pickands dependence function is given by

$$
A(t)=\frac{\log \tilde{C}\left(y^{1-t}, y^{t}\right)}{\log y} \text { for all } t \in[0,1] \text { and for any } y \in(0,1) .
$$

Proposition 7. Pickands dependence function for the extreme value copula $\tilde{C}^{(a)}$ from (21) is given by

$$
A(t)= \begin{cases}1-t+\frac{1}{a} \cdot(1-t)^{\frac{1-a}{2}} \cdot t^{\frac{1+a}{2}} & \text { if } 0 \leq t \leq \frac{1}{2}, \\ t+\frac{1}{a} \cdot t^{\frac{1-a}{2}} \cdot(1-t)^{\frac{1+a}{2}} & \text { if } \frac{1}{2} \leq t \leq 1,\end{cases}
$$

whereas for the extreme value copula $\tilde{C}^{(a)}$ from (20), is simply $A(t)=1$ for $t \in[0,1]$. 
Proof. One has to apply formula (34) for the extreme value copulas (20) and (21).

Figure 7 depicts Pickands dependence function for some values of $a$ for the extreme value copulas $\tilde{C}^{(a)}$ from Proposition 4. Pickands depenence function also allows an alternative way to quantify the distance between $\Pi$ and $\tilde{C}^{(a)}$ (see also Trutschnig et al [20]). Namely,

$$
\operatorname{dist}_{\tilde{C}^{(a)}, \Pi}:=1-2 \cdot \int_{0}^{1 / 2} A(t) d t .
$$

Note that this definition amounts to 0.25 as the largest possible distance between an extreme value copula and the independence copula. The distance between the corresponding $C^{(a)}$ and $\Pi$ is also shown in Figure 7.

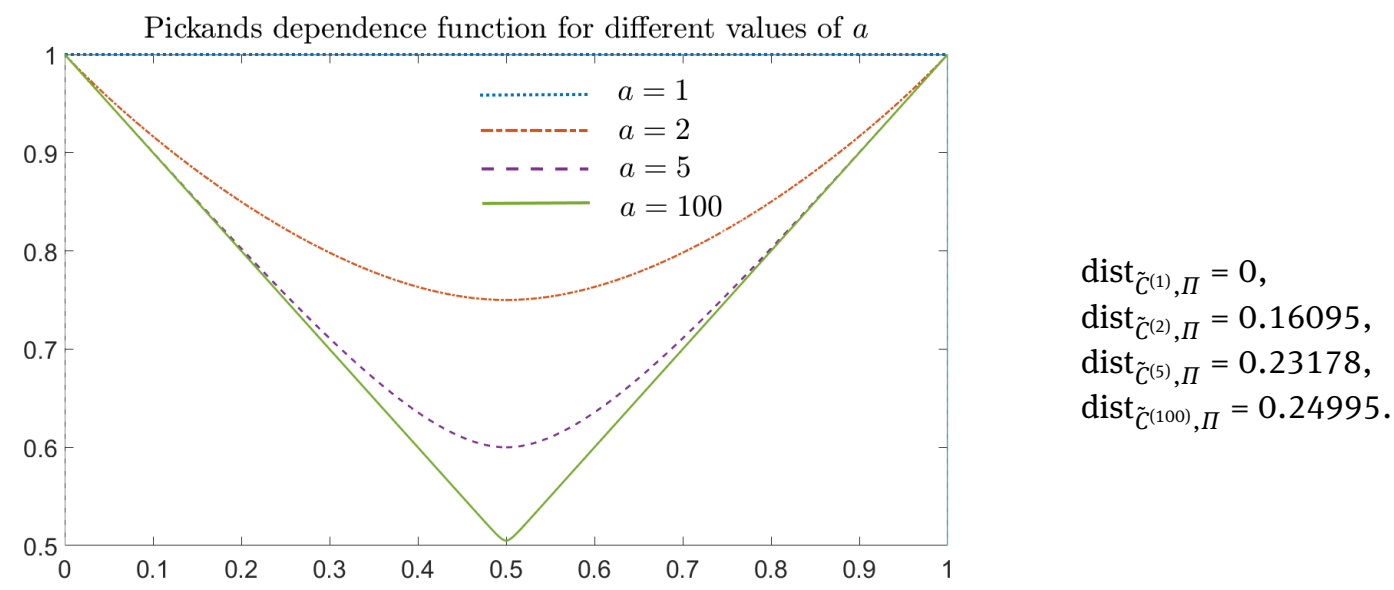

Figure 7

Proposition 8. Let $a \geq 1$. For the new extreme-value copula (21), the lower and upper tail dependence coefficients are

$$
\lambda_{L}=0 \quad \text { and } \quad \lambda_{U}=\frac{a-1}{a}
$$

Proof. Elementary computation, using Definition 1 and formula (21).

\section{Computations in the multivariate $\operatorname{Freund}(a, n)$ model}

We draw the reader's attention that several approaches and models can be found in the literature, which generalize Freund's bivariate model, or where Freund's bivariate model appears as a special case. We mention Shaked et al. [17] and Norros [15] as two examples. On the other hand, our focus is different from that of the authors who have already contributed to this issue, so in this sense we believe that the multivariate setting that we propose is novel.

\subsection{The $n$-variate one-step model with uniform cascading parameter}

We consider our model with $n$ institutions facing a one-step cascade, where the lifetime variables are governed by a matrix $A$ according to the following. The diagonal elements $\lambda_{1}, \ldots, \lambda_{n} \in \mathbb{R}^{+}$are the initial lifetime 
intensities of the institutions, i.e., $Y_{j} \sim \operatorname{Exp}\left(\lambda_{j}\right),(j=1, \ldots, n)$ are independent of each other. Each nondiagonal element $a_{i, j}(i \neq j, i, j=1, \ldots, n)$ expresses the effect of the default of institution $i$ on institution $j$, by adding the value $a_{i, j} \geq 0$ to the original lifetime parameter $\lambda_{j}$. It can be formulated as

$$
X_{j, n}= \begin{cases}\min \left\{Y_{1}, \ldots, Y_{n}\right\}, & \text { if } \min \left\{Y_{1}, \ldots, Y_{n}\right\}=Y_{j}, \\ \min \left\{Y_{1}, \ldots, Y_{n}\right\}+Z_{j}, \text { where } Z_{j} \sim \operatorname{Exp}\left(\lambda_{j}+a_{i, j}\right), & \text { if } \min \left\{Y_{1}, \ldots, Y_{n}\right\}=Y_{i} \neq Y_{j},\end{cases}
$$

for $j=1, \ldots, n$, where $Z_{j}$ s are independent of each other and of all $Y_{i}(i=1, \ldots, n)$. The notation emphasizes that the distribution of the variables depends on $n$. The above mechanism defines an exchangable multivariate distribution, in notation $\underline{X}_{n}:=\left(X_{1, n}, \ldots X_{i, n}, \ldots, X_{n, n}\right) \sim \operatorname{Freund}(A)$. We set $\lambda_{1}=\ldots=\lambda_{n}=1$ and $a_{i, j}=a \geq 0$ in the entire Section 3, and we will use the notation $\underline{X}_{n} \sim \operatorname{Freund}(a, n)$. (For the generalization of Proposition 9 see Appendix A.) The model also allows for the case $a=\infty$. In this case, $X_{1, n}=\ldots=X_{n, n}$ with probability one. In the following, we present the joint density function, the marginal densities, and the marginal cdfs of the Freund $(a, n)$ distribution.

Proposition 9. Let $\underline{X}_{n}$ be Freund $(a, n)$ distributed, where $a \in[0, \infty)$.

(i) The joint pdf of $\underline{X}_{n}=\left(X_{1, n}, \ldots X_{i, n}, \ldots, X_{n, n}\right)$ is given by

$$
f_{\underline{X}_{n}}\left(x_{1}, \ldots, x_{n}\right)=(1+a)^{n-1} \cdot \exp \left\{-\sum_{i=1}^{n} x_{i}-a \cdot \sum_{i=1}^{n}\left(x_{i}-\min _{k=1, \ldots, n} x_{k}\right)\right\}, \quad x_{1}, \ldots, x_{n} \geq 0 .
$$

(ii) If $n \neq a+1$, then any one-dimensional marginal density, i.e., the pdf of each $X_{i, n}$, is given by

$$
f_{X_{i, n}}(x)=-\frac{n a}{n-a-1} \cdot e^{-n x}+\frac{(1+a)(n-1)}{n-a-1} \cdot e^{-(1+a) x}, \quad x \geq 0 .
$$

(iii) If $n \neq a+1$, then any one-dimensional marginal cdf, i.e., the cdf of each $X_{i, n}$, is given by

$$
F_{X_{i, n}}(x)=1+\frac{a}{n-a-1} \cdot e^{-n x}-\frac{n-1}{n-a-1} \cdot e^{-(1+a) x}, \quad x \geq 0 .
$$

Proof. To see (i), one has to compute

$$
\lim _{\Delta x_{1}, \ldots, \Delta x_{n} \rightarrow 0} \frac{\mathbb{P}\left(X_{1, n} \in\left[x_{1}, x_{1}+\Delta x_{1}\right], \ldots, X_{n, n} \in\left[x_{n}, x_{n}+\Delta x_{n}\right]\right)}{\Delta x_{1} \cdot \ldots \cdot \Delta x_{n}} .
$$

This can be done in an elementary way and results in (36). Assertion (ii) can be verified similarly; (iii) follows from (ii) by integration.

\section{Remark 6.}

(i) For $a=0$, the marginal density reduces to $f_{X_{i, n}}(x)=e^{-x}$ and the joint density reduces to $f_{\underline{X}_{n}}\left(x_{1}, \ldots, x_{n}\right)=$ $-\exp \left\{\sum_{i=1}^{n} x_{i}\right\}$, which is obvious, since in this case, the marginals are independent and $\operatorname{Exp}(1)$ distributed.

(ii) For $a=\infty$, the marginal density is given by $f_{X_{i, n}}(x)=n e^{-n x}$. This is obvious, since in this case $X_{1, n}=$ $\ldots=X_{n, n} \sim \operatorname{Exp}(n)$. This formula can be also attained by taking the limit $a \rightarrow \infty$ in (37). The joint distribution of $\left(X_{1, n}, \ldots, X_{n, n}\right)$ is degenerate and concentrated on a one-dimensional subset in $\mathbb{R}^{n}$, namely on $x_{1}=\ldots=x_{n}$.

(iii) For $n \rightarrow \infty$, the marginal density reduces to $f_{X_{i, \infty}}(x)=(1+a) e^{-(1+a) x}$, which can be understood as follows. In case $n \gg 1$, the lifetime variables are nearly independent of each other: one institute receives the shock, and the others (i.e., all except the one who received the shock) essentially do not depend on each other any more.

(iv) Based on the marginal density (37), for $a>0$, the distribution of $X_{i, n}$ can be seen as a generalized mixture (see Definition 5)

$$
X_{i, n}=\mathcal{S}\left(w_{0}, w_{1} ; G_{0}, G_{1}\right),
$$


where $G_{0} \sim \operatorname{Exp}(n), G_{1} \sim \operatorname{Exp}(1+a), w_{0}=\frac{-a}{n-a-1}, w_{1}=\frac{n-1}{n-a-1}$, so $w_{0}+w_{1}=1$, and either $w_{0}$ or $w_{1}$ is non-positive.

(v) If $n=a+1$, then the one-dimensional marginal densities and cdfs can be computed similarly as in the proof of Proposition 9, and they are given by

$f_{X_{i, n}}(x)=(1+n(n-1) x) \cdot e^{-n x} \cdot \mathbb{1}_{\{x \geq 0\}}$, and $F_{X_{i, n}}(x)=\left[1-(1+(n-1) x) \cdot e^{-n x}\right] \cdot \mathbb{1}_{\{x \geq 0\}} \cdot$

Definition 5. We say that a random variable $Z$ is a generalized mixture of the random variables $Z_{1}, \ldots, Z_{n}$, if for their pdfs $f_{Z}, f_{Z_{1}}, \ldots, f_{Z_{n}}$ the relation

$$
f_{Z}=\sum_{i=1}^{n} w_{i} \cdot f_{Z_{i}}
$$

holds, where $\sum_{i=1}^{n} w_{i}=1$. Notice that this definition allows that some of the weights are negative. We use the notation

$$
Z=\mathcal{S}\left(w_{1}, \ldots, w_{n} ; Z_{1}, \ldots, Z_{n}\right)
$$

Remark 7. Notice that (40) only gives a representation of the pdf of the random variable $Z$ in terms of the pdfs of $Z_{1}, \ldots, Z_{n}$, but it does not provide a probabilistic model. In other words, having the random variables $Z_{1}, \ldots, Z_{n}$ given by their pdfs $f_{Z_{1}}, \ldots, f_{Z_{n}}$, we are not aware of any straightforward manner in general for constructing a random variable $Z$ whose pdf is $f_{Z}$. Hence (40) is only a reformulation of the relation (39) among the density functions.

\subsubsection{Expectation and variance of the marginal distributions}

Some standard characteristics of the one-dimensional marginal distributions, for instance the expectation, the second moment, and the variance are easy to determine by using (37) and computing the suitable integrals. They will be derived again in Subsusbsection 3.2.2 more elegantly and more generally. For all $1 \leq i \leq n$, we have

$$
\begin{gathered}
\mathbb{E}\left(X_{i, n}\right)=\frac{n+a}{(a+1) n}, \\
\mathbb{E}\left(X_{i, n}^{2}\right)=\frac{-2 a}{n^{2}(n-a-1)}+\frac{2(n-1)}{(1+a)^{2}(n-a-1)}=\frac{2}{(a+1)^{2}}+\frac{2 a(a+n+1)}{(a+1)^{2} n^{2}}, \\
\mathbb{V}\left(X_{i, n}\right)=\frac{-2 a}{n^{2}(n-a-1)}+\frac{2(n-1)}{(1+a)^{2}(n-a-1)}-\frac{(n+a)^{2}}{(1+a)^{2} n^{2}}=\frac{1}{(a+1)^{2}}+\frac{a(a+2)}{(a+1)^{2} n^{2}} .
\end{gathered}
$$

One immediately sees that for any fixed $0 \leq a<\infty$,

$$
\mathbb{E}\left(X_{i, n}\right)=\frac{1}{1+a}+O(1 / n) \text { and } \mathbb{V}\left(X_{i, n}\right)=\frac{1}{(1+a)^{2}}+O\left(1 / n^{2}\right) \text { as } n \rightarrow \infty .
$$

Notice that for $a=0$, the above characteristics do not depend on $n$ : in particular, $\mathbb{E}\left(X_{i, n}\right)=1, \mathbb{E}\left(X_{i, n}^{2}\right)=2$, and $\mathbb{V}\left(X_{i, n}\right)=1$ for all $n$ (cf. Remark 6 (i)).

\subsubsection{The $k$-dimensional marginal distributions}

It is trivial due to exchangeability that the law of $\left(X_{i_{1}, n}, \ldots, X_{i_{k}, n}\right)$ does not depend on the choice of the indices, so when we aim to determine its distribution, then we can assume without loss of generality that $i_{j}=j$ for $j=1, \ldots, k,(1 \leq k \leq n)$. 
Proposition 10. For $0 \leq a<\infty$, if $n \neq k(1+a)$, then the $k$-dimensional marginal density, i.e., the pdf of $\left(X_{1, n}, \ldots, X_{k, n}\right)$, is given by

$$
\begin{aligned}
& f_{k, n}\left(x_{1}, \ldots, x_{k}\right)=(1+a)^{k-1} \cdot \exp \left\{-n \cdot \min _{1 \leq i \leq k} x_{i}-(1+a)\left(\sum_{i=1}^{k} x_{i}-k \cdot \min _{1 \leq i \leq k} x_{i}\right)\right\}+ \\
& +\frac{(1+a)^{k} \cdot(n-k)}{n-k(1+a)} \cdot \exp \left\{-(1+a) \cdot \sum_{i=1}^{k} x_{i}\right\} \cdot\left(1-\exp \left\{-(n-k(1+a)) \cdot \min _{1 \leq i \leq k} x_{i}\right\}\right) .
\end{aligned}
$$

Proof. In order to derive (44), we did not integrate the joint density (36) $n-k$ times, which would also be possible. We applied model-based considerations, i.e., we elaborated the probability $\mathbb{P}\left(X_{1, n} \in\left[x_{1}, x_{1}+\Delta x_{1}\right], \ldots, X_{k, n} \in\left[x_{k}, x_{k}+\Delta x_{k}\right]\right)$ in an elementary way, similar to the proof of Proposition 9.

\subsubsection{Correlation between the components}

We provide the special case of formula (44) for $k=2$. Then we will use it to compute the covariance and correlation of $X_{i, n}$ and $X_{j, n}$. It is given by

$$
\begin{aligned}
f_{2, n}(x, y) & =(1+a) \cdot \exp \{-(a+1) \cdot \max \{x, y\}-(n-a-1) \cdot \min \{x, y\}\}+ \\
& +\frac{(1+a)^{2} \cdot(n-2)}{n-2-2 a} \cdot \exp \{-(1+a)(x+y)\} \cdot(1-\exp \{-(n-2-2 a) \cdot \min \{x, y\}\}) .
\end{aligned}
$$

From (45), it follows by integration that

$$
\mathbb{E}\left(X_{i, n} \cdot X_{j, n}\right)=\frac{1}{(n-2 a-2)} \cdot\left(\frac{2 a(a+1)(3 n-2 a-2)}{n^{2}(n-a-1)^{2}}+\frac{n-2}{(1+a)^{2}}-\frac{2 a n}{(n-a-1)^{2}(a+1)}\right) .
$$

From (41), (43), and (46), the correlation can be computed:

$$
\operatorname{corr}\left(X_{i, n}, X_{j, n}\right)=\frac{P(a, n)}{Q(a, n)},
$$

where $P(a, n)$ and $Q(a, n)$ are polynomials of $a$ and $n$ with $\operatorname{deg} P=\operatorname{deg} Q=5$, namely

$$
\begin{gathered}
P(a, n)=2 a(a+1)\left[(a+1)^{2}(3 n-2 a-2)+n^{3}\right]+(n-a-1)^{2}\left[3 a^{2} n+4 a n+2 a^{3}+2 a^{2}\right], \\
Q(a, n)=(n-2 a-2)(n-a-1)\left[-2 a(a+1)^{2}+2(n-1) n^{2}-(n+a)^{2}(n-a-1)\right] .
\end{gathered}
$$

It is easy to see that for $0 \leq a<\infty, \operatorname{corr}\left(X_{i, n}, X_{j, n}\right) \underset{n \rightarrow \infty}{\longrightarrow} 0, \quad$ and the rate of convergence is $1 / n^{2}$, i.e., $\lim _{n \rightarrow \infty} n^{2} \cdot \operatorname{corr}\left(X_{i, n}, X_{j, n}\right)=l$, with $0<l<\infty$.

Observe that for $a=0$, the correlation is zero for all $n$ (cf. Remark 6 (i)).

\subsection{Distributions of sums of components in the $n$-variate one-step model - Exponential Gamma Mixture Type I distribution}

Our aim in the following is to investigate the average lifetime of the entities in the system for fixed $n$ and for $n \rightarrow \infty$. Let us introduce the following notation:

$$
\begin{gathered}
S_{n}:=X_{1, n}+\ldots+X_{n, n} \\
S_{k, n}:=X_{1, n}+\ldots+X_{k, n} \text { for } k=1, \ldots, n,
\end{gathered}
$$

i.e., $S_{n}=S_{n, n}$. We provide an explicit form and an integral form for the pdf of $S_{k, n}$. (Due to exchangeability, $S_{k, n}$ could also be defined by $X_{i_{1}, n}+\ldots+X_{i_{k}, n}$ with any $k$-element subset of $\{1, \ldots, n\}$.) 


\subsubsection{Straightforward derivation and the explicit form of the pdf of $S_{k, n}$}

Proposition 11. For any fixed $n$ and for all $k=1, \ldots, n$, if $n \neq k \cdot(1+a)$, then the probability density function of $S_{k, n}$ is given by

$$
g_{S_{k, n}}(s)=c(a, n, k) \cdot e^{-\frac{n s}{k}}+e^{-(1+a) s} \cdot \operatorname{pol}_{k-1}(s), \quad \text { for } s \geq 0,
$$

where

$$
\operatorname{pol}_{k-1}(s)=\sum_{j=0}^{k-1} c_{j}(a, k, n) \cdot s^{j}
$$

with

$$
\left.\begin{array}{c}
c(a, n, k)=\frac{(-1)^{k} k^{k-1} n a(a+1)^{k-1}}{(n-k(1+a))^{k}}, \\
c_{j}(a, n, k)=\frac{(-1)^{k-j-1} \cdot k^{k-j-1}}{j !} \cdot \frac{n a(1+a)^{k-1}}{(n-k(1+a))^{k-j}} \text { for } j=0, \ldots, k-2, \\
c_{k-1}(a, n, k)=\frac{1}{(k-1) !} \cdot \frac{(n-k)(1+a)^{k}}{n-k(1+a)} .
\end{array}\right\}
$$

In particular, when $k=n$, if $a \neq 0$, then

$$
g_{S_{n, n}}(s)=\left(1+\frac{1}{a}\right)^{n-1} \cdot\left[e^{-s}-\left(\sum_{j=0}^{n-2} \frac{1}{j !} a^{j} s^{j}\right) \cdot e^{-(1+a) s}\right], \quad s \geq 0 .
$$

Proof. The proof is a straightforward, but sometimes extremely cumbersome computation. It is clear that

$$
g_{S_{k, n}}(s)=\int_{H_{k}} f_{k, n}\left(s_{1}, \ldots, s_{k}\right) d s_{1} \ldots d s_{k-1},
$$

where $H_{k}=\left\{\left(s_{1}, \ldots, s_{k}\right): s_{1}+\ldots+s_{k}=s\right\}$. From (44), it is clear that $f_{k, n}$ is a linear combination of exponential functions, where the exponents are linear combinations of the (integration) variables $s_{1}, \ldots, s_{k}$. Furthermore, the integration region $H_{k}$ translates to linear expressions concerning the integration bounds if the $(k-1)$-dimensional integral in (51) is written out as $k-1$ (successive) one-dimensional integral. It means that in the $j$-th integration step $(j=1, \ldots, k-1)$, the current integrand consists of a pure exponential term and an exponential multiplied by a polynomial of degree $j-1$. Due to the above-mentioned facts (regarding $f_{k, n}$ and the linear expressions in the integration bounds) the result of the $j$-th integration $\operatorname{step}(j=1, \ldots, k-1)$ is a function consisting of a pure exponential term and an exponential multiplied by a polynomial of degree $j$. This argumentation already explains the structure given in (48). We show the computation in reasonable details for $k=2$ in Appendix C, and we will also indicate how the proof can be carried out for $k>2$.

Remark 8. According to (49), the value of $c(0, n, n), c_{0}(0, n, n)$ and $c_{n-1}(0, n, n)$ might be ambiguous. By evaluating in the correct order, namely starting with $a=0$ followed by $k=n$, one gets $c(0, n, n)=$ $c_{0}(0, n, n)=0$ and $c_{n-1}(0, n, n)=\frac{1}{(n-1) !}$. This little technicality will become important in Subsection 3.3.

Remark 9. Observe the following.

(i) If $a=\infty$ for any fixed $k$ and $n$, then

$$
g_{S_{k, n}(s)}=\frac{n}{k} \cdot e^{-\frac{n s}{k}}, \quad \operatorname{Exp}(n / k) \text { distribution } .
$$

(ii) If $n \rightarrow \infty$ for any fixed $a$ and $k$, then

$$
\lim _{n \rightarrow \infty} g_{S_{k, n}(s)}=\frac{1}{(k-1) !} \cdot e^{-(1+a) s} \cdot(1+a)^{k} \cdot s^{k-1}, \quad \operatorname{Gamma}(k, a+1) \text { distribution, }
$$


where we use the following parametrization of the gamma distirbution: gamma_pdf $f_{\alpha, \beta}(s)=\frac{\beta^{\alpha}}{\Gamma(\alpha)} s^{\alpha-1} e^{-\beta s}$ for $s \geq 0$.

So $S_{k, n}$ is, roughly speaking, the sum of $k$ almost independent $\operatorname{Exp}(1+a)$ variables for large $n$, i.e., when $n \gg k$; and it can be seen as the sum of $k$, indeed independent $\operatorname{Exp}(1+a)$ variables for the limiting case $n \rightarrow \infty$ (cf. Remark 6 (iii)).

(iii) If $a=0$ for any fixed $k$ and $n$, then

$$
g_{S_{k, n}(s)}=\frac{1}{(k-1) !} \cdot e^{-s} \cdot s^{k-1}, \quad \operatorname{Gamma}(k, 1) \text { distribution } .
$$

Remark 10. Based on Proposition 11, for $1 \leq k<n$, the variable $S_{k, n}$ can be seen as a generalized mixture of an exponential distribution and $k$ gamma distributions, one of which is in fact an exponential distribution. The case $k=n$ is slightly different: $S_{n, n}$ is a generalized mixture of an exponential and $n-1$ gamma distributions, one of which is in fact an exponential distribution. We formulate this assertion in the next statement.

Proposition 12. Let the random variable $G_{0}$ be $\operatorname{Exp}(n / k)$ distributed and the random variables $G_{j}$ be $\operatorname{Gamma}(j, 1+a)$ distributed $(j=1, \ldots, k)$. Assume that $n \neq k(1+a)$.

Then $S_{k, n}$ can be written as

$$
S_{k, n}=\mathcal{S}\left(w_{0}, \ldots, w_{k} ; G_{0}, \ldots G_{k}\right) \text {, }
$$

where the mixing weights $w_{j}$ are

$$
\begin{gathered}
w_{0}=\frac{(-1)^{k} \cdot k^{k} a(1+a)^{k-1}}{(n-k(1+a))^{k}}, \\
w_{j}=\frac{(-1)^{k-j} \cdot k^{k-j} n a(1+a)^{k-j-1}}{(n-k(1+a))^{k-j+1}} j=1, \ldots, k-1, \\
w_{k}=\frac{n-k}{n-k(1+a)} .
\end{gathered}
$$

Proof. The assertion follows from Proposition 11 by identifying the pdfs of the $\operatorname{Exp}(n / k)$ and $\operatorname{Gamma}(j, 1+a)$ distributions. $\sum_{j=0}^{k} w_{j}=1$ can be seen by a cumbersome, but elementary computation. Notice that the mixture is indeed a generalized mixture: $w_{j}<0$ if $k-j$ is odd and $n-k(1+a)>0$; and $w_{j}<0$ for all $j=1, \ldots, k$ if $n-k(1+a)<0$.

\subsubsection{Model based derivation and the integral form of the pdf of $S_{k, n}$}

Due to the definition of the multivariate model given in (35), $S_{k, n}$ can be rewritten as

$$
S_{k, n}=E_{k, n}+\left(Z^{(k-1)}\right)^{I} \cdot\left(Z^{(k)}\right)^{1-I},
$$

where $E_{k, n}:=k \cdot \min \left\{Y_{1}, \ldots, Y_{n}\right\}$ is $\operatorname{Exp}(n / k)$ distributed (or equivalently, $\operatorname{Gamma}(1, n / k)$ distributed), and $Z^{(m)}:=\sum_{j=1}^{m} Z_{j}$, where $Z_{j}$ s are i.i.d. $\operatorname{Exp}(1+a)$, therefore $Z^{(m)}(m=k-1, k)$ follows a $\operatorname{Gamma}(m, a+1)$ distribution, $I$ is the indicator of the event that $\min \left\{Y_{1}, \ldots, Y_{n}\right\}$ is attained on one of $Y_{1}, \ldots, Y_{k}$, furthermore $E_{k, n}$ and $Z^{(m)}$ are independent of each other. With the use of (54), in order to derive the pdf of $S_{k, n}$, one only needs to compute the convolutions of the densities of a $\operatorname{Gamma}(1, n / k)$ and a $\operatorname{Gamma}(m, 1+a)$ distribution $(m=k-1, k)$, and weighting them with $\mathbb{P}(I=1)=\frac{k}{n}$ and $\mathbb{P}(I=0)=\frac{n-k}{n}$, which proves Proposition 13. In particular, if $k=n$, then $\mathbb{P}(I=1)=1$, which means that

$$
S_{n, n}=E_{n, n}+Z^{(n-1)},
$$

where $E_{n, n} \sim \operatorname{Exp}(1)$ and $Z^{(n-1)} \sim \operatorname{Gamma}(n-1, a+1)$ are independent of each other. 
Proposition 13. The integral form of the pdf of $S_{k, n}$ for $k=1, \ldots, n$ is given by

$$
g_{S_{k, n}}(s)=e^{-\frac{n s}{k}} \cdot \frac{(1+a)^{k-1}}{k !} \cdot \int_{0}^{s} e^{-(1+a) x} \cdot\left(k(k-1) x^{k-2}+(1+a)(n-k) x^{k-1}\right) d x
$$

In particular,

$$
g_{S_{n, n}}(s)=e^{-s} \cdot \frac{(1+a)^{n-1}}{(n-2) !} \cdot \int_{0}^{s} e^{-(1+a) x} \cdot x^{n-2} d x
$$

Remark 11. Notice that (54) can be rewritten equivalently as

$$
S_{k, n}=E_{k, n}+G_{k-1}+Z_{k}^{\star} \text {, }
$$

where the summands are independent, $E_{k, n} \sim \operatorname{Exp}(n / k), G_{k-1} \sim \operatorname{Gamma}(k-1, a+1)$, and $Z_{k}^{\star}=\left\{\begin{array}{cl}0 & \text { with probability } \frac{k}{n}, \\ Z_{k} \sim \operatorname{Exp}(a+1) & \text { with probability } \frac{n-k}{n} .\end{array}\right.$

Using (58), we can derive elegantly the expectation and variance of $S_{k, n}$. We have

$$
\begin{gathered}
\mathbb{E}\left(S_{k, n}\right)=\mathbb{E}\left(E_{k, n}\right)+\mathbb{E}\left(G_{k-1}\right)+\mathbb{E}\left(Z_{k}^{\star}\right)=\frac{k}{n}+\frac{k-1}{a+1}+\frac{n-k}{n} \cdot \frac{1}{a+1}=\frac{k \cdot(a+n)}{n \cdot(a+1)}, \\
\mathbb{V}\left(S_{k, n}\right)=\mathbb{V}\left(E_{k, n}\right)+\mathbb{V}\left(G_{k-1}\right)+\mathbb{V}\left(Z_{k}^{\star}\right)=\frac{k^{2}}{n^{2}}+\frac{k-1}{(a+1)^{2}}+\frac{n^{2}-k^{2}}{n^{2}(1+a)^{2}}=\frac{k}{(a+1)^{2}}+\frac{k^{2}}{n^{2}} \cdot \frac{a(a+2)}{(a+1)^{2}} .
\end{gathered}
$$

Definition 6. The Exponential Gamma Mixture Type I (in notation $E G M(n, k, a)$ or EGM Type I) distribution is defined by its probability density function via the formulas (48)-(49), or alternatively by formula (56).

The first approach (formulas (48)-(49)) gives an algebraic way for defining the $E G M(n, k, a)$ distribution, as it was pointed out in Proposition 12, while the second approach (decomposition (58)) gives a probabilistic way. The pdf of the $\operatorname{EGM}(n, k, a)$ distribution for some $n, k, a$ values is plotted in Figure 8 .

Probability density functions of the

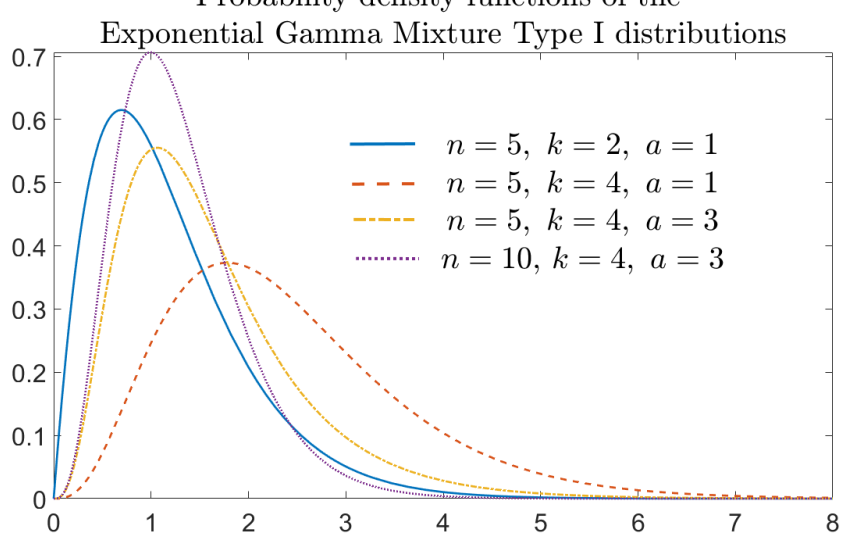

Figure 8

Remark 12. For fixed $k$ and for $n \rightarrow \infty$, the weights $w_{j}(j=0, \ldots, k-1)$ in (53) vanish, while $w_{k} \rightarrow 1$, so the generalized mixture in (52) reduces to a $\operatorname{Gamma}(k, a+1)$ distribution, as it was already stated in Remark 9 (ii), i.e., the $\operatorname{EGM}(\infty, k, a)$ distribution is identical to the $\operatorname{Gamma}(k, a+1)$ distribution. 


\subsubsection{Asymptotics for $S_{n, n}$ : gamma approximation}

Since no simple, well tractable formula for the pdf of $S_{n, n}$ is available (see (50) and (57)), we aim to understand the asymptotical behaviour of $S_{n, n}$ by suitably approximating its pdf. We provide an asymptotics which arises from modifying a lower bound for the density $g_{S_{n, n}}$.

Setting $k=n$ in (59) and (60) yields

$$
\begin{gathered}
\mathbb{E}\left(S_{n, n}\right)=\frac{n+a}{1+a}, \\
\mathbb{V}\left(S_{n, n}\right)=\frac{n}{(1+a)^{2}}+\frac{a(a+2)}{(a+1)^{2}} .
\end{gathered}
$$

Proposition 14. For all $s \geq 0$ and for all $n \in \mathbb{N}$, it holds that

$$
\frac{e^{-(1+a) s}(a+1)^{n-1} s^{n-1}}{(n-1) !} \leq g_{S_{n, n}}(s) \leq \frac{e^{-s}(a+1)^{n-1} s^{n-1}}{(n-1) !} .
$$

Proof. The Taylor polynomial of degree $n-2$ of the function $e^{x}$ centered at the origin is $T_{n-2}(x)=\sum_{j=0}^{n-2} \frac{1}{j !} x^{j}$. The function value $e^{a s}$ can be approximated by $e^{a s} \approx \sum_{j=0}^{n-2} \frac{1}{j !} a^{j} s^{j}$, and the Lagrange remainder term is $\frac{e^{\xi}}{(n-1) !}(a s)^{n-1}$ for some $\xi \in[0, a s]$. Therefore the error committed by the approximation can be lower and upper bounded by

$$
\frac{(a s)^{n-1}}{(n-1) !}=\min _{\xi \in[0, a s]} \frac{e^{\xi}(a s)^{n-1}}{(n-1) !} \leq \frac{e^{\xi}(a s)^{n-1}}{(n-1) !} \leq \max _{\xi \in[0, a s]} \frac{e^{\xi}(a s)^{n-1}}{(n-1) !}=\frac{e^{a s}(a s)^{n-1}}{(n-1) !} .
$$

Inserting these bounds into (50), the statement follows.

Remark 13. Formulas (61) and (62) show that the distribution of $S_{n, n}$ is degenerate in the sense that $\mathbb{E}\left(S_{n, n}\right) \rightarrow \infty$ and $\mathbb{V}\left(S_{n, n}\right) \rightarrow \infty$ as $n \rightarrow \infty$, but it is still worth examining its distribution as we will see in the following. The upper bound in (63) (or alternatively the integral form (57)) shows that $\lim _{n \rightarrow \infty} g_{S_{n, n}}(s)=0$ for all $s \geq 0$. When the lower bound is multiplied by $(a+1)$, it turns into the pdf of a $\operatorname{Gamma}(n, a+1)$ distribution, which can be used to approximate the density $g_{S_{n, n}}$ after appropriate scaling and shifting. This assertion is formulated precisely in the next statement and illustrated in Figure 9. (For $n=100$ the difference between the curves is hardly any visible.)

Proposition 15. Let us define the random variables $\tilde{Y}_{n}=\beta_{n} \cdot Y_{n}+\alpha_{n}$, where $Y_{n} \sim \operatorname{Gamma}(n, a+1), \beta_{n}=\sqrt{\frac{n+a(a+2)}{n}}, \alpha_{n}=\frac{1}{1+a}\left(n \cdot\left(1-\beta_{n}\right)+a\right)$. Let us denote the probability density function of $\tilde{Y}_{n}$ by $\tilde{g}_{n}$. Then

$$
\lim _{n \rightarrow \infty} \int_{-\infty}^{\infty}\left|g_{S_{n, n}}(s)-\tilde{g}_{n}(s)\right| d s=0
$$

We call $\tilde{Y}_{n}$ the gamma approximation of $S_{n, n}$, although $\tilde{Y}_{n}$ (due to the $\alpha_{n}$ term) is not gamma-distributed, but follows a shifted gamma distribution, which justifies the terminology. The proof can be found in Appendix B. 
The pdfs of $S_{30}, S_{50}, S_{100}$ and their gamma-approximations for $a=2$

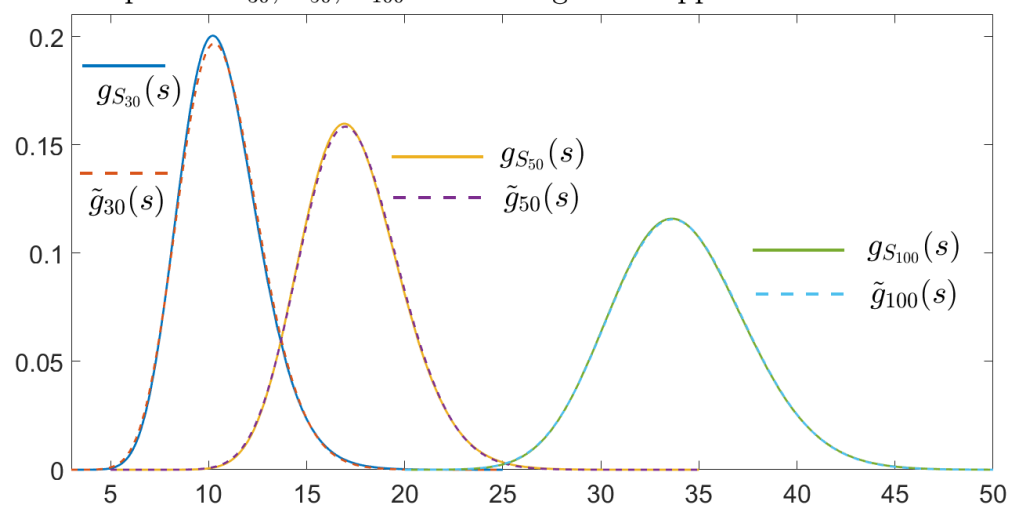

Figure 9: Probability density functions of the sum of the components and their gamma approximations for $n=30,50,100$. The $L_{1}$-distances are $\left\|g_{S_{30}}-\tilde{g}_{30}\right\| \approx 0.0254,\left\|g_{S_{50}}-\tilde{g}_{50}\right\| \approx 0.0141,\left\|g_{S_{100}}-\tilde{g}_{100}\right\| \approx 0.0059$. The mode of $\tilde{Y}_{n}$ is $\frac{n+a-\beta_{n}}{a+1}=$ $\frac{n+a-1}{a+1}+O(1 / n)$, so the same asymptotics holds for the mode of $S_{n, n}$.

Remark 14. It is easy to verify that the $L_{1}$-distance of pdf functions is invariant under monotone transformations of the underlying random variables. So Proposition 15 means that $\lim _{n \rightarrow \infty}\left\|g_{T\left(S_{n, n}\right)}-g_{T\left(\tilde{Y}_{n}\right)}\right\|_{L_{1}}=0$, where $T$ is an arbitrary monote transformation, and $g_{T\left(S_{n, n}\right)}$ and $g_{T\left(\tilde{Y}_{n}\right)}$ are the corresponding density functions.

\subsection{Distribution of $\bar{X}_{n}$, asymptotics and limiting distribution - Exponential Gamma Mixture Type II distribution}

Let us introduce the notation

$$
\bar{X}_{n}:=\frac{X_{1, n}+\ldots+X_{n, n}}{n} .
$$

In order to obtain a non-trivial limiting distribution for $\bar{X}_{n}$, we need to compute $\mathbb{E}\left(\bar{X}_{n}\right)$ and $\mathbb{D}\left(\bar{X}_{n}\right)$. Using (41) (or equivalently (61)) and (62), we get trivially that

$$
\begin{gathered}
\mathbb{E}\left(\bar{X}_{n}\right)=\frac{n+a}{(a+1) n}, \\
\mathbb{V}\left(\bar{X}_{n}\right)=\frac{1}{n^{2}} \mathbb{V}\left(S_{n, n}\right)=\frac{1}{n(1+a)^{2}}+\frac{a(a+2)}{n^{2}(a+1)^{2}} .
\end{gathered}
$$

\section{Remark 15.}

(i) If $a=0$, then $\mathbb{V}\left(\bar{X}_{n}\right)=\frac{1}{n}$ for all $n$.

(ii)

$$
\text { For any fixed } 0 \leq a<\infty, \mathbb{V}\left(\bar{X}_{n}\right)=\frac{1}{n(1+a)^{2}}+o(1 / n) \text { as } n \rightarrow \infty .
$$

(iii) For all fixed $n, \mathbb{V}\left(\bar{X}_{n}\right) \underset{a \rightarrow \infty}{\longrightarrow} \frac{1}{n^{2}}$ (cf. $\bar{X}_{n}=\min _{1 \leq i \leq n}\left\{X_{i, n}\right\} \sim \operatorname{Exp}(n)$ for $\left.a=\infty\right)$.

Formulas (65) and (67) provide the suitable normalizing terms for deriving a limiting distribution for $\bar{X}_{n}$. The rescaled variable has mean zero and variance one:

$$
\bar{X}_{n}^{0}:=\frac{\bar{X}_{n}-\mathbb{E}\left(\bar{X}_{n}\right)}{\mathbb{D}\left(\bar{X}_{n}\right)} .
$$




\subsubsection{The distribution of $\bar{X}_{n}^{0}$ and its limiting distribution}

The probabilty density function of $\bar{X}_{n}^{0}$ can be derived using the probability density function $g_{S_{n, n}}(s)$ given in (50).

$$
g_{\bar{X}_{n}^{0}}(s)=n \cdot \mathbb{D}\left(\bar{X}_{n}\right) \cdot g_{S_{n, n}}\left(n \cdot \mathbb{D}\left(\bar{X}_{n}\right) \cdot s+n \cdot \mathbb{E}\left(\bar{X}_{n}\right)\right) \quad \text { for all } s \geq-\frac{\mathbb{E}\left(\bar{X}_{n}\right)}{\mathbb{D}\left(\bar{X}_{n}\right)} .
$$

Due to (50), (65) and (67), the following asymptotics holds for $g_{\bar{X}_{n}^{0}}(s)$.

Proposition 16. For all $s \in \mathbb{R}$, we have

$$
\lim _{n \rightarrow \infty} g_{\bar{X}_{n}^{0}}(s)-\frac{\sqrt{n}}{1+a}\left(1+\frac{1}{a}\right)^{n-1} \cdot\left[e^{-\frac{\sqrt{n} s+n+a}{a+1}}-\left(\sum_{j=0}^{n-2} \frac{1}{j !}\left(\frac{a}{a+1}(\sqrt{n} s+n+a)\right)^{j}\right) \cdot e^{-(\sqrt{n} s+n+a)}\right]=0 .
$$

It is remarkable that the approximating expression in (68) defines a two-parametric family of probability density functions with asymptotically the same support as that of $g_{\bar{X}_{n}^{0}}(s)$. We call this family Exponential Gamma Mixture Type II distribution, and to the best of our knowledge, it has not been mentioned in the literature yet.

Definition 7. Let $a>0$. The Exponential Gamma Mixture (EGM) Type II (in notation $E G M(n, a)$ ) distribution is defined by its probability density. For $s \geq-\sqrt{n}-\frac{a}{\sqrt{n}}$,

$$
g_{E G M(n, a)}(s):=\frac{\sqrt{n}}{1+a}\left(1+\frac{1}{a}\right)^{n-1} \cdot\left[e^{-\frac{\sqrt{n} s+n+a}{a+1}}-\left(\sum_{j=0}^{n-2} \frac{1}{j !}\left(\frac{a}{a+1}(\sqrt{n} s+n+a)\right)^{j}\right) \cdot e^{-(\sqrt{n} s+n+a)}\right] .
$$

The pdf of the $\operatorname{EGM}(n, a)$ distribution is pictured in Figure 10 for some values of $a$ and $n$.

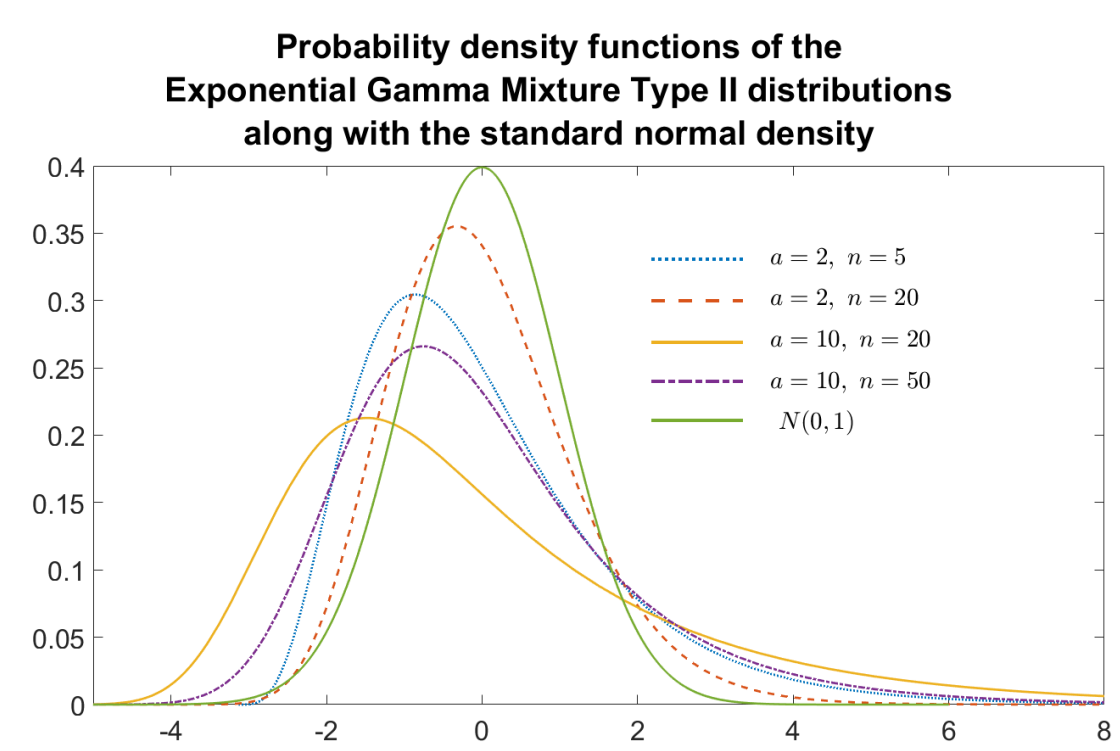

Figure 10: The convergence to the $N(0,1)$ limiting distribution is faster when $a$ is smaller.

Proposition 17. Let $Z$ be $E G M(n, a)$ distributed.

Then $Z$ is a generalized mixture of a shifted exponential distribution and $n-1$ shifted gamma distributions, with

$$
Z=\mathcal{S}\left(w_{E}, w_{0}, \ldots w_{n-2} ; E, \tilde{G}_{0}, \ldots \tilde{G}_{n-2}\right) \text {, }
$$


where $E \sim \operatorname{Exp}(\sqrt{n} /(a+1))-\frac{n+a}{\sqrt{n}}$ and $\tilde{G}_{j} \sim \operatorname{Gamma}(j+1, \sqrt{n})-\frac{n+a}{\sqrt{n}}, j=0, \ldots n-2$, and the mixing weights are

$$
w_{E}=\left(\frac{a+1}{a}\right)^{n-1}, \quad w_{j}=-\frac{1}{1+a}\left(\frac{a+1}{a}\right)^{n-j-1}, j=0, \ldots, n-2 .
$$

Proof. One has to identify the suitable pdfs in (68) and has to verify that the weights indeed sum up to one.

Remark 16. The mixture in (70) is indeed a generalized mixture: the weights satisfy $w_{j}<0$ for $j=0, \ldots, n-2$ and they are unbounded as $n \rightarrow \infty$.

Finally, we state the limiting distribution of the $\operatorname{EGM}(n, a)$ distribution. The core idea is the following: although the variables $X_{1, n}, \ldots, X_{n, n}$ are not independent, the dependence among them vanishes as $n \rightarrow \infty$ (cf. Remark 6). The statement is illustrated in Figure 10.

Proposition 18. Assume that $a>0$ and let $Z_{n} \sim \operatorname{EGM}(n, a)$. Then

$$
\lim _{n \rightarrow \infty} \mathbb{P}\left(Z_{n} \leq x\right)=\Phi(x),
$$

where $\Phi$ is the cdf of the standard normal distribution.

Proof. We will show that $\bar{X}_{n}^{0}$ is asymptotically $N(0,1)$ distributed, which proves the statement in the light of Proposition 16. Let us consider decomposition (55) again:

$$
S_{n, n}=E_{n, n}+\sum_{j=1}^{n-1} Z_{j},
$$

where $E_{n, n} \sim \operatorname{Exp}(n), Z_{j} \sim \operatorname{Exp}(1+a)(j=1, \ldots, n-1)$ are independent. This decomposition enables us to provide a relation between $\bar{X}_{n}^{0}$ and $\bar{Z}_{n-1}^{0}:=\frac{\bar{Z}_{n-1}-\mathbb{E}\left(\bar{Z}_{n-1}\right)}{\mathbb{D}\left(\bar{Z}_{n-1}\right)}$, where $\bar{Z}_{n-1}=\frac{1}{n-1} \sum_{j=1}^{n} Z_{j}$. Namely,

$$
\bar{X}_{n}^{0}=\frac{\bar{X}_{n}-\mathbb{E}\left(\bar{X}_{n}\right)}{\mathbb{D}\left(\bar{X}_{n}\right)}=\frac{a+1}{\sqrt{n+a(a+2)}} \cdot E_{n, n}^{0}+\sqrt{\frac{n-1}{n+a(a+2)}} \cdot \bar{Z}_{n-1}^{0},
$$

where $E_{n, n}^{0}=\frac{E_{n, n}-\mathbb{E}\left(E_{n, n}\right)}{\mathbb{D}\left(E_{n, n}\right)}$. The variable $\frac{a+1}{\sqrt{n+a(a+2)}} \cdot E_{n, n}^{0}$ has zero mean and its variance converges to zero as $n \rightarrow \infty$. The coefficient of $\bar{Z}_{n-1}^{0}$ in (71) converges to one, and the limiting distribution of $\bar{Z}_{n-1}^{0}$ is $N(0,1)$ by the central limit theorem, hence the same holds true for $\bar{X}_{n}^{0}$.

Proposition 19. Let $\tilde{\tilde{Y}}_{n}=\frac{1+a}{\sqrt{n}} \cdot Y_{n}-\sqrt{n}$, where $Y_{n} \sim \operatorname{Gamma}(n, a+1)$, and let $g_{\tilde{Y}_{n}}$ its pdf. Then $\lim _{n \rightarrow \infty} \| g_{\bar{X}_{n}^{0}}-$ $g_{\tilde{Y}_{n}} \|_{L_{1}}=0$.

Proof. $\bar{X}_{n}^{0}$ can be written as as $\bar{X}_{n}^{0}=T\left(S_{n, n}\right)$, and $\tilde{Y}_{n}$ can be written as $\tilde{Y}_{n}=T\left(\tilde{Y}_{n}\right)$, where $T(x)=\frac{1+a}{\sqrt{n+a(a+2)}} \cdot x-$ $\frac{n+a}{\sqrt{n+a(a+2)}}$ is a monotone increasing transformation. Hence, in the light of Remark 14, according to Proposition 15 the statement holds.

Remark 17. With the help of the variable $\tilde{Y}_{n}=\frac{1+a}{\sqrt{n}} \cdot Y_{n}-\sqrt{n}$, where $Y_{n} \sim \operatorname{Gamma}(n, a+1)$, one can derive another proof for the fact that $\bar{X}_{n}^{0}$ is asymptotically $N(0,1)$ distributed. We will show that for all $s \in \mathbb{R}$, $\lim _{n \rightarrow \infty} g_{\tilde{\tilde{Y}}_{n}}(s)=\frac{1}{\sqrt{2 \pi}} e^{-s^{2} / 2}$

Notice that $g_{\tilde{Y}_{n}}(s)=\frac{\sqrt{n}}{1+a} \cdot g a m m a \_p d f_{n, a+1}\left(\frac{\sqrt{n} s+n}{1+a}\right)=\frac{\sqrt{n}}{(n-1) !} \cdot e^{-(\sqrt{n} s+n)} \cdot(\sqrt{n} s+n)^{n-1}$. Using Stirling's formula for $(n-1)$ !, we get

$$
\lim _{n \rightarrow \infty} g_{\tilde{\tilde{Y}}_{n}}(s)=\lim _{n \rightarrow \infty} \frac{1}{\sqrt{2 \pi}} \cdot e^{-(\sqrt{n} s+1)} \cdot\left(\frac{\sqrt{n} s+n}{n-1}\right)^{n-1} .
$$


Using the second order Taylor expansion of $\log (1+x)$, one can write

$$
\begin{aligned}
& \log \left(\frac{\sqrt{n} s+n}{n-1}\right)^{n-1}=(n-1) \cdot \log \left(1+\frac{\sqrt{n} s+1}{n-1}\right)=(n-1) \cdot\left(\frac{\sqrt{n} s+1}{n-1}-\frac{1}{2}\left(\frac{\sqrt{n} s+1}{n-1}\right)^{2}+O\left(n^{-3 / 2}\right)\right)= \\
& =\sqrt{n} s+1-\frac{1}{2} s^{2}+O\left(n^{-1 / 2}\right) . \text { Inserting this into the right-hand-side of }(72) \text {, the statement follows. }
\end{aligned}
$$

\section{Outlook}

We have seen that the model introduced by Freund [4], and elaborated further by Guzmics and Pflug [6] still contains many interesting and as yet unexplored details, concerning extreme value questions and multivariate generalizations. In a future work, we plan to obtain results for the componentwise minimum, analogous to those we presented for the maximum in this current work. We also guess that the analysis presented in Section 2 and Section 3 can be also performed in the general Freund $(A)$ model, though it would be even more technical, see Appendix A. Neveretheless, that would highly increase the applicability of the model for real-life situations, as well as a detailed elaboration of a multi-step cascade. Furthermore, we would like to extend our examinations that we performed so far to stochastic order relations, too.

\section{Appendix A}

In Subsection 3.1, we dealt with a cascading matrix, where all cascading parameters $a_{i, j}(i \neq j)$ were the same. Let us now consider the general case, i.e., when $A=\left\{a_{i, j}\right\}_{i, j=1}^{n}$, where $a_{i, j} \geq 0$ for $i \neq j$, and the diagonal elements are the initial lifetime intensities $\lambda_{i}(i=1, \ldots, n)$.

The joint probability density function of the corresponding lifetime variables $\left(X_{1,1}, \ldots, X_{1, n}\right)$ is given by

$$
f_{A}\left(x_{1}, \ldots, x_{n}\right)=\lambda_{i} \cdot\left(\prod_{\substack{j=1 \\
j \neq i}}^{n}\left(\lambda_{j}+a_{i, j}\right)\right) \cdot \exp \left\{-\underline{\lambda}^{\top} \underline{x}-\underline{e}_{i}^{\top} A\left(\underline{x}-x_{i} \mathbb{1}\right)\right\} \begin{array}{r}
\text { for } x_{1}, \ldots, x_{n} \geq 0, \\
x_{i}=\min _{1 \leq k \leq n} x_{k},
\end{array}
$$

where $\underline{\lambda}^{\top}=\left(\lambda_{1}, \ldots, \lambda_{n}\right), \underline{x}^{\top}=\left(x_{1}, \ldots, x_{n}\right), \mathbb{1}^{\top}=(1, \ldots, 1)$, and $\underline{e}_{i}^{\top}=\left(0, \ldots 0, \begin{array}{c}i \text {-th } \\ \text { entry }\end{array}, \ldots, 0\right)$.

To derive (73), one has to elaborate $\mathbb{P}\left(X_{1, n} \in\left[x_{1}, x_{1}+\Delta x_{1}\right], \ldots, X_{n, n} \in\left[x_{n}, x_{n}+\Delta x_{n}\right]\right)$ under the assumption that $x_{i}=\min _{1 \leq k \leq n} x_{k}$. It can be done in an elementary way. By setting $\underline{\lambda}=\mathbb{1}$ and $a_{i, j}=a \geq 0$ for $i \neq j$, one obtains (36), which was presented in Subsection 3.1.

\section{Appendix B}

\section{Proof of Proposition 15.}

(I) The support of $\tilde{g}_{n}(s)$ is $\left(\alpha_{n}, \infty\right)$ and the support of $g_{S_{n, n}}(s)$ is $(0, \infty)$. Note that $\alpha_{n} \downarrow \frac{-a^{2}}{2(1+a)}<0$ for $a>0$. Therefore first we split the integral into two parts.

$$
\begin{gathered}
\int_{\alpha_{n}}^{0}\left|g_{S_{n, n}}(s)-\tilde{g}_{n}(s)\right| d s=\mathbb{P}\left(\tilde{Y}_{n}<0\right)=\mathbb{P}\left(Y_{n}<\frac{-\alpha_{n}}{\beta_{n}}\right)=\int_{0}^{-\frac{\alpha_{n}}{\beta_{n}}} \frac{(a+1)^{n} s^{n-1} e^{-(a+1) s}}{(n-1) !} d s \leq \\
\leq \int_{0}^{\frac{a^{2}}{2(1+a)}} \frac{(a+1)^{n} s^{n-1} e^{-(a+1) s}}{(n-1) !} d s \leq e^{-\frac{a^{2}}{2}}\left(\frac{a^{2}}{2}\right)^{n} \cdot \frac{1}{(n-1) !} \longrightarrow 0 \text { as } n \rightarrow \infty .
\end{gathered}
$$


(II) The integral $\int_{0}^{\infty}\left|g_{S_{n, n}}(s)-\tilde{g}_{n}(s)\right| d s$ will be devided again into two parts.

(II A) Introducing the notation $m_{n}:=\mathbb{E}\left(S_{n, n}\right)=\frac{n+a}{1+a}$ and $\sigma_{n}:=\frac{\sqrt{n}}{1+a} \approx \mathbb{D}\left(S_{n, n}\right)$ (see (61) and (62)), we have that

$$
\begin{gathered}
\int_{\sigma_{n} \cdot \sqrt{n}+m_{n}}^{\infty}\left|g_{S_{n, n}}(s)-\tilde{g}_{n}(s)\right| d s \leq \int_{\sigma_{n} \cdot \sqrt{n}+m_{n}}^{\infty} g_{S_{n, n}}(s) d s+\int_{\sigma_{n} \cdot \sqrt{n}+m_{n}}^{\infty} \tilde{g}_{n}(s) d s \stackrel{(\star)}{=} \\
=\mathbb{P}\left(S_{n, n} \geq \sigma_{n} \cdot \sqrt{n}+m_{n}\right)+\mathbb{P}\left(\tilde{Y}_{n} \geq \sigma_{n} \cdot \sqrt{n}+m_{n}\right) \leq \mathbb{P}\left(\left|S_{n, n}-m_{n}\right| \geq \sigma_{n} \cdot \sqrt{n}\right)+ \\
+\mathbb{P}\left(\left|\tilde{Y}_{n}-\tilde{m}_{n}\right| \geq \tilde{\sigma}_{n} \cdot(\sqrt{n}+O(1 / \sqrt{n}))\right) \leq \frac{1}{n}+\frac{1}{n}=\frac{2}{n} \longrightarrow 0 \quad \text { as } n \rightarrow \infty .
\end{gathered}
$$

At $\left(^{\star}\right)$ we took it into account that $\tilde{m}_{n}:=\mathbb{E}\left(\tilde{Y}_{n}\right)=m_{n}$ and $\tilde{\sigma}_{n}:=\mathbb{D}\left(\tilde{Y}_{n}\right)=\sigma_{n}+O(1 / \sqrt{n})$, and then we used Chebyshev's inequality.

(II B) It remains to show that

$$
\lim _{n \rightarrow \infty} \int_{0}^{\sigma_{n} \cdot \sqrt{n}+m_{n}}\left|g_{S_{n, n}}(s)-\tilde{g}_{n}(s)\right| d s=\lim _{n \rightarrow \infty} \int_{0}^{\frac{2 n+a}{1+a}} g_{S_{n, n}}(s)-\tilde{g}_{n}(s) \mid d s=0 .
$$

It happens through the following steps.

Step 1: One can verify that

$$
\tilde{g}_{n}(s)=\frac{a+1}{\beta_{n}}\left(\int_{0}^{s}\left(\tilde{g}_{n-1}(x)-\tilde{g}_{n}(x)\right) d x\right)+R_{n},
$$

where $R_{n}=\left(\frac{a+1}{\beta_{n}}\right)^{n} \cdot \frac{1}{(n-1) !} e^{-(a+1) \frac{\alpha_{n}}{\beta_{n}}}+\frac{a+1}{\beta_{n}}\left(\int_{\alpha_{n-1}}^{0} \tilde{g}_{n-1}(x) d x-\int_{\alpha_{n}}^{0} \tilde{g}_{n}(x) d x\right)$ can be handled with the same technique that was used in (I). Note that $R_{n}=o(1 / n)$, which is important because the length of the integration interval in (74) is $\frac{2 n+a}{1+a}$.

Step 2: Using (57) and (75), we can write

$$
\begin{aligned}
\tilde{g}_{n}(s) & -g_{S_{n, n}}(s)=\int_{0}^{s}\left(\frac{a+1}{\beta_{n}}\right)^{n} \cdot \frac{1}{(n-2) !}\left(x-\alpha_{n}\right)^{n-2} \cdot e^{-(a+1) \frac{x-\alpha_{n}}{\beta_{n}}} \cdot\left[1-\frac{1}{\beta_{n}} \cdot \frac{a+1}{n-1} \cdot\left(x-\alpha_{n}\right)\right] d x \\
& -e^{-s} \cdot \frac{(1+a)^{n-1}}{(n-2) !} \int_{0}^{s} e^{-(1+a) x} x^{n-2} d x+R_{n} .
\end{aligned}
$$

Step 3: By substituting $y=\frac{x-\alpha_{n}}{\beta_{n}}$ in the first integral, and simply replacing $x$ with $y$ in the second one, we obtain

$$
\begin{aligned}
\tilde{g}_{n}(s) & -g_{S_{n, n}}(s)=\int_{-\alpha_{n} / \beta_{n}}^{\left(s-\alpha_{n}\right) / \beta_{n}} \frac{(a+1)^{n}}{\beta_{n}} \cdot \frac{1}{(n-2) !} y^{n-2} \cdot e^{-(a+1) y} \cdot\left[1-\frac{a+1}{n-1} \cdot y\right] d y \\
& -e^{-s} \cdot \frac{(1+a)^{n-1}}{(n-2) !} \int_{0}^{s} e^{-(1+a) y} y^{n-2} d y+R_{n} .
\end{aligned}
$$

Note that the intersection of the two integration intervals is $\left[-\alpha_{n} / \beta_{n}, s\right]$ if $n$ is large enough. The nonoverlapping parts have to be handled separately (see Step 4(a) and Step 4(b)).

\section{Step 4(a):}

$$
\int_{0}^{\frac{2 n+a}{1+a}} e^{-s} \cdot \frac{(1+a)^{n-1}}{(n-2) !} \int_{0}^{-\alpha_{n} / \beta_{n}} e^{-(1+a) y} y^{n-2} d y d s \leq \int_{0}^{\frac{2 n+a}{1+a}} e^{-s} \cdot \frac{(1+a)}{(n-2) !} e^{-(n-2)}(n-2)^{n-2} \frac{-\alpha_{n}}{\beta_{n}} d s \leq O(1 / \sqrt{n})
$$


where in the first step we used that the maximum of $e^{-(1+a) y} y^{n-2}$ is attained at $\frac{n-2}{a+1}$, and in the second step we performed the integral and applied Stirling's formula for the factorial.

Step 4(b):

$$
\int_{0}^{\frac{2 n+a}{1+a}} \int_{s}^{\left(s-\alpha_{n}\right) / \beta_{n}} \frac{(a+1)^{n}}{\beta_{n}} \cdot \frac{1}{(n-2) !} y^{n-2} \cdot e^{-(a+1) y} \cdot\left[1-\frac{a+1}{n-1} \cdot y\right] d y d s \leq O\left(\left(\frac{2}{e}\right)^{n} \cdot \frac{1}{n^{3 / 2}}\right)
$$

can be seen similarly as Step 4(a). Here it has to be taken into account that the maximum of $y^{n-2} \cdot e^{-(a+1) y}$ on interval $\left[s,\left(s-\alpha_{n}\right) / \beta_{n}\right]$ is attained at the right endpoint of the interval.

Step 4(c): Now we focus on that part of (74), which was not considered yet.

$$
\begin{aligned}
I_{n}:=\int_{0}^{\frac{2 n+a}{1+a}}\left|\int_{-\alpha_{n} / \beta_{n}}^{s} \frac{(a+1)^{n-1}}{(n-2) !} y^{n-2} \cdot e^{-(a+1) y} \cdot\left[\frac{1}{\beta_{n}}\left(1-\frac{a+1}{n-1}\right) \cdot y-e^{-s}\right] d y\right| d s \leq \\
\quad \leq \int_{0}^{\frac{2 n+a}{1+a}} \frac{1}{(n-2) !} \underbrace{\left|\int_{0}^{(a+1) s} e^{-z} \cdot\left(c_{n} \cdot z-e^{-s}\right) \cdot z^{n-2} d z\right|}_{g(s):=} d s=: B_{n},
\end{aligned}
$$

where the upper bound $B_{n}$ was obtained by increasing the integration interval, and substituting $z=(a+1) y$, and where $c_{n}=\frac{1}{\beta_{n}}\left(\frac{1}{a+1}-\frac{1}{n-1}\right) \rightarrow \frac{1}{1+a}$ as $n \rightarrow \infty$. Performing the inner integral one sees that $g(s)=O\left(e^{-s(a+1)} s^{n-1}(a+1)^{n-1}\right)$.

Since the primitive function of $e^{-s(a+1)} s^{n-1}(a+1)^{n-1}$ is also of the same type, namely $e^{-s(a+1)} \cdot \operatorname{pol}_{n-1}(s)$. $(a+1)^{n-2}$, where $\operatorname{pol}_{n-1}(s)$ is a polynomial of degree $n-1$, we can write that

$$
B_{n} \leq \text { const } \cdot\left[\frac{1}{(n-2) !} e^{-s(a+1)} s^{n-1}(a+1)^{n-2}\right]_{0}^{\frac{2 n+a}{1+a}} .
$$

Substituting the bounds, and applying Stirling’s formula yields

$$
I_{n} \leq B_{n} \leq O\left(\sqrt{n}\left(\frac{2}{e}\right)^{n}\right),
$$

which finally proves the statment.

\section{Appendix C}

We provide the proof of Proposition 11 for $k=2$ and the sketch of the proof for $k>2$. The density $g_{S_{2, n}}(s)$ can be written as

$$
\begin{aligned}
g_{S_{2, n}}(s) & =\int_{0}^{s} f_{2, n}(t, s-t) d t \stackrel{(\star)}{=} \int_{0}^{s / 2} f_{2, n}(t, s-t) d t+\int_{s / 2}^{s} f_{2, n}(t, s-t) d t=2 \cdot \int_{0}^{s / 2} f_{2, n}(t, s-t) d t= \\
& =2 \cdot \int_{0}^{s / 2}(1+a) e^{-(a+1) s-(n-2 a-2) t}+\frac{(1+a)^{2}(n-2)}{n-2-2 a} \cdot e^{-(1+a) s} \cdot\left(1-e^{-(n-2-2 a) t}\right) d t= \\
& =\frac{2 n a(a+1)}{(n-2-2 a)^{2}} \cdot e^{-\frac{n s}{2}}+e^{-(1+a) s} \cdot\left(\frac{-2 n a(1+a)}{(n-2-2 a)^{2}}+\frac{(n-2)(1+a)^{2}}{n-2-2 a} \cdot s\right), \text { as it was claimed. }
\end{aligned}
$$


At $\left.{ }^{\star}\right)$ we split the integral into two parts according to (44), i.e., we take it into account that the minimum is attained in which variable. Then we use the symmetry with respect to the variables.

For $k \geq 3$ one can work in a similar way (integration by parts). Meanwhile one can observe and conjecture the sample in the sequence of coefficients (across the terms for a fixed $k$, and also across the terms for different $k$ values), and finally the formulas given in (49) can be verified.

\section{Appendix D}

As it was mentioned in Remark 1, due to the courtesy of an anonymous referee, Proposition 1 is a direct consequence of a much more general but simple fact: Suppose that $C$ is an absolutely continuous copula whose density $c$ is square integrable on $[0, \delta]^{2}$ for some $\delta \in(0,1)$. Then the lower tail dependence coefficient of $C$ is 0 .

Proof: Considering $u \in(0, \delta)$ and applying Cauchy-Bunyakovsky-Schwarz inequality yields

$$
\frac{C(u, u)}{u}=\frac{1}{u} \int_{[0, u]^{2}} c(x, y) d x d y \leq \frac{1}{u} u\|c\|_{2,[0, u]^{2}}=\|c\|_{2,[0, u]^{2}},
$$

where $\|c\|_{2,[0, u]^{2}}$ denotes the $L^{2}$-norm of $c$ on $[0, u]^{2}$. Considering that $\lim _{u \rightarrow 0+}\|c\|_{2,[0, u]^{2}}=0$ (by dominated convergence) the result follows.

It was discussed in [6], Section 2.3, that the copula density stemming from $(7)$ is bounded around $(0,0)$, therefore also square integrable on $[0, \delta]^{2}$. So the above result applies to our case and provides an alternative proof for Proposition 1.

\section{References}

[1] de Haan, L. and S. I. Resnick (1977). Limit theory for multivariate sample extremes. Z. Wahrscheinlichkeitstheor. verw. Geb. 40(4), 317-337.

[2] Devroye, L. (1986). Non-Uniform Random Variate Generation. Springer, New York.

[3] Drees, H. and X. Huang (1998). Best attainable rates of convergence for estimators of the stable tail dependence function. J. Multivariate Anal. 64(1), 25-47.

[4] Freund, J. E. (1961). A bivariate extension of the exponential distribution. J. Amer. Statist. Assoc. 56(296), 971-977.

[5] Gudendorf, G. and J. Segers (2010). Extreme-value copulas. In P. Jaworski, F. Durante, W. K. Härdle, and T. Rychlik (Eds.), Copula Theory and Its Applications, pp. 127-145. Springer, Heidelberg.

[6] Guzmics, S. and G. Pflug (2019). Modelling cascading effects for systemic risk: properties of the Freund copula. Depend. Model. 7, 24-44.

[7] Hochrainer-Stigler, S., J. Balkovic, K. Silm, and A. Timonina-Farkas (2019). Large scale extreme risk assessment using copulas: an application to drought events under climate change for Austria. Comput. Manag. Sci. 16(4), 651-669.

[8] Jongman, B., S. Hochrainer-Stigler, L. Feyen, J.C.J.H Aerts, R. Mechler, W.J.W. Botzen, L.M. Bouwer, G. Pflug, R. Rojas, and P.J. Ward (2014). Increasing stress on disaster-risk finance due to large floods. Nat. Clim. Chang. 4(4), 264-268.

[9] Leadbetter, M. R., G. Lindgren, and H. Rootzén (1983). Extremes and Related Properties of Random Sequences and Processes. Springer, New York.

[10] Mai, J.-F. and M. Scherer (2017). Simulating Copulas: Stochastic Models, Sampling Algorithms, and Applications. Second edition. World Scientific, Singapore.

[11] Marshall, A. W. and I. Olkin (1967). A multivariate exponential distribution. J. Amer. Statist. Assoc. 62, 30-44.

[12] Marshall, A. W. and I. Olkin (1988). Families of multivariate distributions. J. Amer. Statist. Assoc. 83(403), 834-841.

[13] Mathieu, R. and S. Mohammed (2013). Extreme value copulas and max-stable processes. J. SFdS. 154(1), 138-150.

[14] Nelsen, R. B. (2006). An Introduction to Copulas. Second edition. Springer, New York.

[15] Norros, I. (1985). Systems weakend by failures. Stochastic Process. Appl. 20(2), 181-196.

[16] Resnick, S. I. (1987). Extreme Values, Regular Variation and Point Processes. Springer, New York.

[17] Shaked, M., F. Spizzichino, and F. Suter (2002). Nonhomogeneous birth processes and ${ }^{\infty}$-spherical densities, with applications in reliability theory. Probab. Engrg. Inform. Sci. 16(3), 271-288. 
[18] Spivey, M. (2013). The expected maximum of independent and identically distributed exponential random variables. Available at https://mikespivey.wordpress.com/2013/05/13/expectedmaxexponential/.

[19] Sun, R. Lecture notes in probability theory I - lecture 12. Available at https://drive.google.com/file/d/10jiERs14c07eb5060qJ0TfsdR_ah3dW/view.

[20] Trutschnig, W., M. Schreyer, and J. Fernández-Sánchez (2016). Mass distributions of two-dimensional extreme-value copulas and related results. Extremes 19(3), 405-427.

[21] von Neumann, J. (1951). Various techniques used in connection with random digits. J. Res. Nat. Bur. Stand. Appl. Math. Series 3, 36-38. 